\title{
Physiological mechanisms of sustained fumagillin-induced weight loss
}

\author{
Jie An, ${ }^{1}$ Liping Wang, ${ }^{1}$ Michael L. Patnode, ${ }^{2,3}$ Vanessa K. Ridaura, ${ }^{2,3}$ Jonathan M. Haldeman, ${ }^{1}$ \\ Robert D. Stevens, ${ }^{1}$ Olga Ilkayeva, ${ }^{1}$ James R. Bain, ${ }^{1}$ Michael J. Muehlbauer, ${ }^{1}$ Erin L. Glynn, ${ }^{1}$ \\ Steven Thomas, ${ }^{1}$ Deborah Muoio, ${ }^{1}$ Scott A. Summers, ${ }^{1}$ James E. Vath, ${ }^{4}$ Thomas E. Hughes, ${ }^{4}$ \\ Jeffrey I. Gordon, ${ }^{2,3}$ and Christopher B. Newgard' \\ 'Sarah W. Stedman Nutrition and Metabolism Center, Duke Molecular Physiology Institute, Departments of Pharmacology \\ and Cancer Biology and Medicine, Duke University Medical Center, Durham, North Carolina, USA. ${ }^{2}$ Center for Cenome \\ Sciences and Systems Biology and ${ }^{3}$ Center for Gut Microbiome and Nutrition Research, Washington University School of \\ Medicine, St. Louis, Missouri, USA. ${ }^{4}$ Zafgen Inc., Boston, Massachusetts, USA.
}

Current obesity interventions suffer from lack of durable effects and undesirable complications. Fumagillin, an inhibitor of methionine aminopeptidase-2, causes weight loss by reducing food intake, but with effects on weight that are superior to pair-feeding. Here, we show that feeding of rats on a high-fat diet supplemented with fumagillin (HF/FC) suppresses the aggressive feeding observed in pair-fed controls (HF/PF) and alters expression of circadian genes relative to the HF/ PF group. Multiple indices of reduced energy expenditure are observed in HF/FG but not HF/PF rats. HF/FC rats also exhibit changes in gut hormones linked to food intake, increased energy harvest by gut microbiota, and caloric spilling in the urine. Studies in gnotobiotic mice reveal that effects of fumagillin on energy expenditure but not feeding behavior may be mediated by the gut microbiota. In sum, fumagillin engages weight loss-inducing behavioral and physiologic circuits distinct from those activated by simple caloric restriction.

Authorship note: JIG and CBN are co-senior authors.

Conflict of interest: JEV and TEH are employees of Zafgen Inc., and Zafgen provided a portion of the funding that supported this work via a sponsored research contract with Duke University. All studies were performed in the laboratories of CBN at Duke or IIG at Washington University.

Submitted: December 21, 2017 Accepted: January 30, 2018 Published: March 8, 2018

\section{Reference information:} JCI Insight. 2018;3(5):e99453. https://doi.org/10.1172/jci. insight. 99453.

\section{Introduction}

Behavioral interventions for treating the current pandemic of obesity, such as diet and exercise, although health-enhancing, suffer from poor compliance and limited long-term efficacy, in part due to compensatory metabolic adaptations (1). Similarly, responses to currently approved medical therapies are limited, with $30 \%-60 \%$ of patients failing to achieve $5 \%$ weight loss over a 3 -month period of treatment (2). Bariatric surgery has a more sustained effect on body weight and comorbidities, such as type 2 diabetes and cardiovascular disease risk, but is a major surgical procedure with risk of complications, including nutritional deficiencies and anemia (3). For all of these reasons, further research is needed on pharmacologic strategies for inducing sustained weight loss and metabolic health in obese subjects.

Fumagillin is a natural product isolated from Aspergillus fumigatus in 1949 and shown decades later to be an inhibitor of angiogenesis $(4,5)$. Methionine aminopeptidase type 2 (MetAP2) was identified as a molecular target of fumagillin in 1997 (6). Both rodent and human studies indicate that fumagillin and fumagillin-related compounds decrease food intake and hunger. In addition, MetAP2 inhibitors have been hypothesized to decrease angiogenesis in adipose tissue, and this has been suggested to lead to loss of fat mass. Indeed, in mice with diet-induced obesity (DIO), chronic fumagillin administration results in reduced adipose blood vessel density and mass (7). However, this effect also is seen in response to caloric restriction and, thus, could be due to vascular remodeling associated with reductions in fat cell size. MetAP2 inhibitors impact intracellular signaling pathways, notably ERK 1/2, providing one explanation for their observed lipid-lowering effects (8). Fumagillin and fumagillin analogs also have been reported to lower leptin levels in concert with reduced fat mass and to increase levels of FGF21 and adiponectin (9). Nonetheless, the mechanisms by which fumagillin produces sustained lowering of food intake and body weight in both rodents and humans remain ill defined. In the present report, we examine how inclusion of fumagillin in a high-fat (HF) diet fed to Wistar rats causes changes in feeding behavior and energy expenditure compared with pair-fed controls. Our analyses include tests of whether the gut microbiota is involved in mediating these effects. Together, our results unveil fumagillin-regulated behavioral and physiological networks that regulate energy balance. 


\section{Results}

Effect of diet interventions on body weight and food intake. To study the mechanism of weight loss induced by fumagillin, we caused DIO in young (6-week-old) Wistar rats by feeding them a HF diet for 12 weeks. A control group was fed standard chow (SC) for the same time period. At 12 weeks, the HF group had a $157 \%$ $\pm 28 \%$ (mean \pm SD) increase in body weight relative to their starting weight, compared with $116 \% \pm 12 \%$ in the SC group ( $n=82$ in the HF group; $n=26$ in the SC group; $P=1.7 \times 10^{-10}$; Figure $\left.1 \mathrm{~A}\right)$. As expected, the HF group consumed significantly more calories over the 12 -week feeding period per animal compared with the SC group ( $P=0.0001$; Figure 1B).

After this 12-week feeding period, animals were divided into 4 intervention groups, with various studies performed on subgroups of animals at 2, 4 , and 8 weeks after the start of the interventions: (i) groups of $\mathrm{HF}$ rats continued on HF diet ad libitum; (ii) groups of SC rats continued on SC diet ad libitum; (iii) groups of $\mathrm{HF}$ rats switched to a $\mathrm{HF}$ diet containing $80 \mathrm{mg}$ fumagillin/kg of $\mathrm{HF}$ food (HF/FG) (see Methods for details of fumagillin preparation and dosing); and (iv) groups of $\mathrm{HF}$ rats pair-fed on $\mathrm{HF}$ diet (HF/PF) to match the reduced rate of food intake observed in the HF/FG group ( $n=26$ rats/treatment group for $\mathrm{HF}$, $\mathrm{HF} / \mathrm{PF}$, and SC for 2-week treatments; $n=34$ for 2-week HF/FG treatment; $n=13$ for 8-week HF, HF/PF, and SC treatments; $n=17$ for 8 -week HF/FG treatment. Four-week intervention studies, including $n$ values, are summarized later in the sequence of experiments). Supplemental Table 1 (supplemental material available online with this article; https://doi.org/10.1172/jci.insight.99453DS1) provides complete information about diets used in this study.

Animals fed HF or SC diets gained weight at similar rates for the 8-week intervention period $(P=$ 0.38; Figure 1C). In contrast, rats fed the HF/FG diet experienced dramatic and sustained weight loss, with decreases in body weight of $-8.4 \% \pm 1.7 \%$ after 2 weeks, $-10.7 \% \pm 3.1 \%$ after 4 weeks, and $-9.4 \% \pm$ $4.5 \%$ after 8 weeks of intervention, respectively. HF/PF caused a similar initial rate of weight loss as the $\mathrm{HF} / \mathrm{FG}$ diet, but the effect was transient, with reductions in body weight of $-5.9 \% \pm 2.3 \%$ after 2 weeks, $-6.6 \% \pm 3.8 \%$ after 4 weeks, and $-3.3 \% \pm 4.4 \%$ after 8 weeks of intervention (Figure 1C). Data shown in Figure 1C summarize changes in body weight as percent weight loss compared with starting weight, with each rat serving as its own control. Measurement of food intake during the interventions, expressed both as grams of food consumed/rat and $\mathrm{kcal} / \mathrm{g}$ body weight, demonstrated a sharp decrease in food intake in the HF/FG group over the first 2 weeks of intervention, with a gradual return toward the rate of food intake of the HF group in weeks 2-8 (Figure 1, D and E). Calculation of average caloric intake over the full 8 weeks of intervention confirmed that the $\mathrm{HF} / \mathrm{FG}$ and $\mathrm{HF} / \mathrm{PF}$ groups had the same rate of food intake, which - for both groups - was significantly lower than the rate of food intake for the HF group fed ad libitum $(P<0.05)$ (Figure $1 \mathrm{~F})$.

Although we believe that showing weight loss data normalized to each animal's starting weight as in Figure 1 is the most appropriate means of displaying our data, we also provide primary data for weight loss in grams/rat for all groups used in this study in Supplemental Figures 1-3, delineated for the 2-week, 4-week, and 8-week intervention time points. Importantly, a simple sum of grams of weight lost per rat demonstrates a significantly greater weight loss in the HF/FG group compared with the HF/PF group over all 3 intervention time periods (Supplemental Figure 1D, Supplemental Figure 2D, and Supplemental Figure 3D). This was true despite the fact that the HF/FG and HF/PF groups consumed exactly the same amount of food in grams/rat over the full study period (Figure 1D). Finally, we also measured water intake in a group of rats fed on $\mathrm{HF}$ diet for 12 weeks and switched to HF/FG diet for 2 weeks. Water intake stayed constant during the 2 weeks of HF/FG feeding, while food intake and body weight were falling (Supplemental Figures 4, A-C), allowing us to conclude that the weight loss induced by fumagillin was not due to reduced water intake.

Fumagillin reduces adiposity and improves insulin sensitivity. The HF/FG group had significantly reduced size and weight of epididymal white adipose tissue (EWAT) as early as 2 weeks after initiation of treatment relative to the $\mathrm{HF}$ group, while the effect of $\mathrm{HF} / \mathrm{PF}$ was not significant until 8 weeks of intervention (Figure $2 \mathrm{~A}$ ). In contrast, $\mathrm{HF} / \mathrm{PF}$ rats had significantly reduced liver weights at 2 and 8 weeks relative to the HF or HF/FG groups, whereas HF/FG feeding had no effect on this measure at either time point (Figure 2B).

Hyperinsulinemic-euglycemic clamp analysis revealed that insulin sensitivity was improved similarly and significantly in HF/FG- and HF/PF-treated rats compared with the HF group, at both the 2-week and 8-week time points, as reflected by higher glucose infusion rates (GIR) (Figure 2C). These results were confirmed by decreased homeostatic model assessment (HOMA) scores (a factorial of insulin $\times$ glucose measured in the fasted state) in the HF/FG and HF/PF groups relative to the HF group at 8 weeks (Supplemental Table 2). 
A

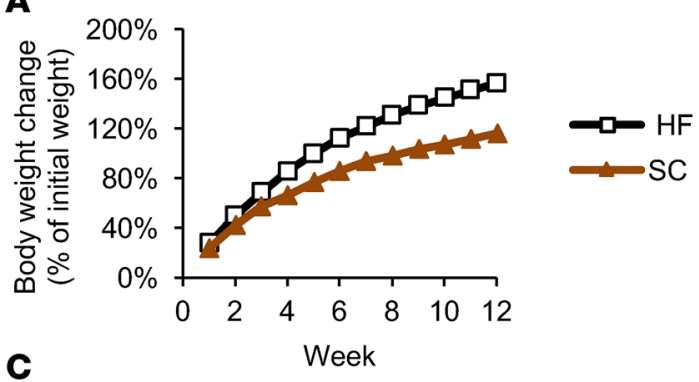

C
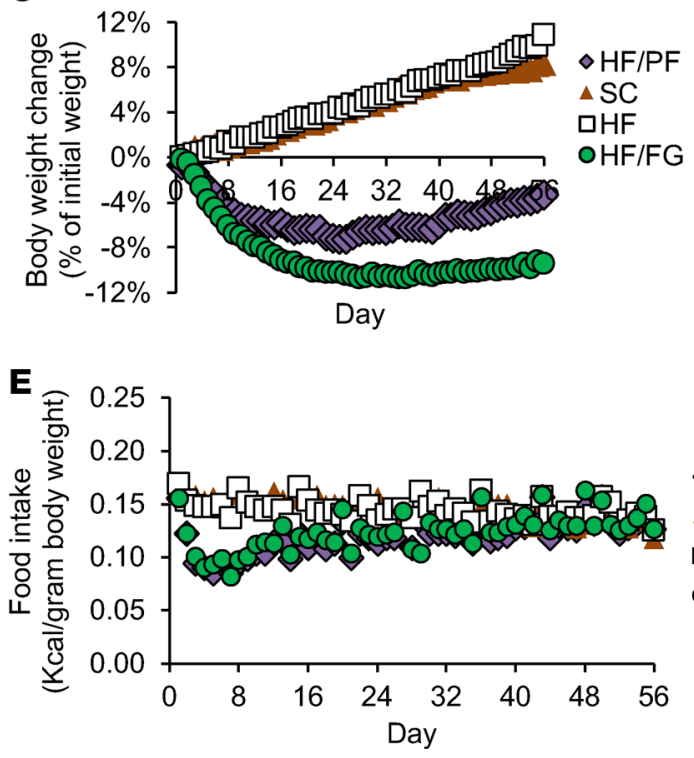

B

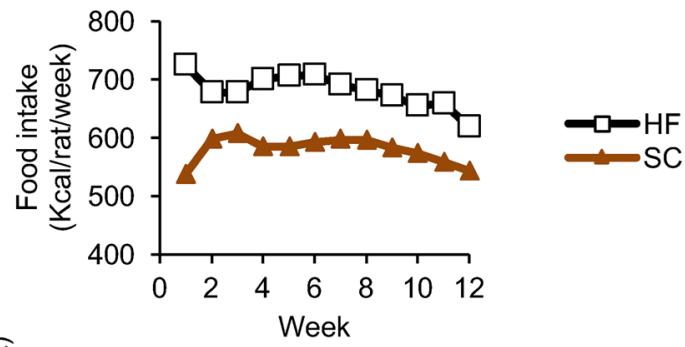

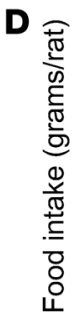
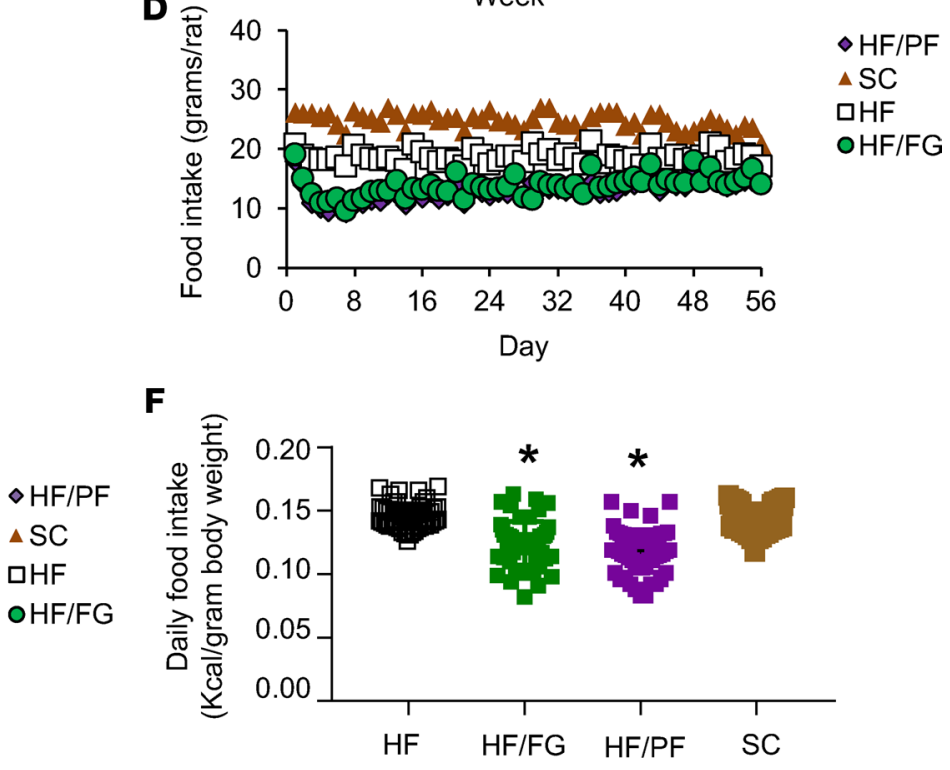

Figure 1. Fumagillin reduces caloric intake and body weight in obese rats. (A) Body weight changes (\% increase relative to starting weight) over the initial 12 weeks of feeding of HF or SC diets. Data are means of $n=86$ for the HF group and $n=26$ for the SC group. Weight gain was significantly different in the HF versus SC animals after 1 week of feeding $(P<0.0012)$. (B) Mean caloric intake over the 12-week feeding period in HF and SC diet groups, expressed as $\mathrm{Kcal} / \mathrm{rat} /$ week. The difference between groups are significant at all time points $(P<0.0004)$. (C) After the initial 12 -week feeding period, animals were divided into 4 intervention groups: (i) HF rats continued on HF diet ad libitum (HF); (ii) SC rats continued on SC diet ad libitum (SC); (iii) HF rats switched to HF/FG diet ad libitum (HF/FG); (iv) HF rats pair-fed on HF diet (HF/PF) to match food intake of the HF/FG group. Body weight change is expressed as percent change relative to the start of the intervention period. Beginning at day 5 of intervention, the HF/FG and HF/PF rats lost weight compared with HF or SC ( $P=0.04-0.00001)$. Data represent $n=26$ for HF, HF/PF, and SC groups at 2 weeks of diet intervention and an $n=13$ for these groups between 2-8 weeks of intervention. For HF/FG, $n=34$ at 2 weeks of diet intervention and $n=17$ from 2-8 weeks. (D) Mean daily food intake in the 4 intervention groups described in $\mathbf{C}$ expressed as grams of food consumed/rat. (E) Daily caloric intake for the groups described in C, expressed as Kcal/gram body weight/ day. The HF and SC groups and the HF/FG and HF/PF groups were not different at any time point. Consumption of HF and HF/FG diets were significantly different $(P<0.024)$ from day $2-38$, except for days $13,19,20,27,32,36$, and 37 . Consumption of HF food by the HF and HF/PF groups were significantly different $(P<0.015)$ from day 1-38, except for days 20, 27, 32, 36, and 37. (F) Mean daily caloric intake, Kcal/gram body weight/day, for groups described in C. Data are mean $\pm \mathrm{SD}$, with ${ }^{*} P<0.05$ when compared with either HF or SC group. Two-tailed, unpaired $t$ tests were performed in all panels. $P<0.05$ with a Bonferroni correction was used to define statistical significance among groups.

Fumagillin suppresses energy expenditure and alters feeding behavior. To begin to assess factors that may influence the differential effects of HF/FG and HF/PF on body weight, we measured energy expenditure and physical activity in metabolic cages. Food was provided to all groups of animals once daily at approximately $1 \mathrm{pm}, 6$ hours after the start of the 12-hour light cycle (Zeitgeber time [ZT] 6:00). Striking differences in metabolic cage variables were observed in the HF/FG versus HF/PF groups at both 2 and 8 weeks of intervention. Animals in the HF/PF group ate their food quickly and aggressively shortly after it was placed in the cage. This was reflected by sharp spikes in oxygen exchange rate $\left(\mathrm{VO}_{2}\right.$, volume of oxygen consumed), physical activity, heat production, and respiratory exchange ratio (RER) that occurred shortly after provision of food (Figures 3 and 4). In contrast, HF/FG-treated rats did not respond to provision of food in the same fashion and exhibited no sudden changes in $\mathrm{VO}_{2}$, physical activity, heat production, or RER. Visual inspection of rats in the 2 groups revealed a nonchalant approach of the HF/FG-treated rats to feeding following meal provision, compared with a more excited and immediate response of the HF/PF group. 
A

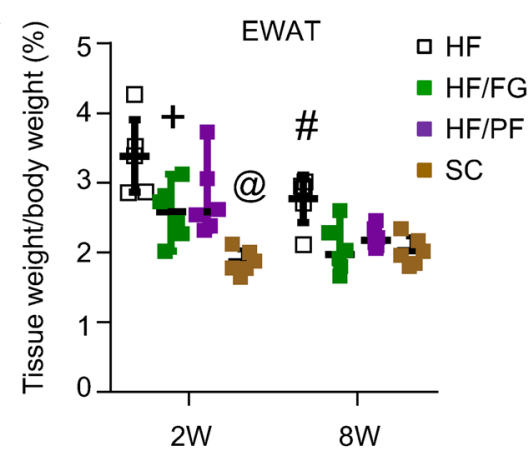

B

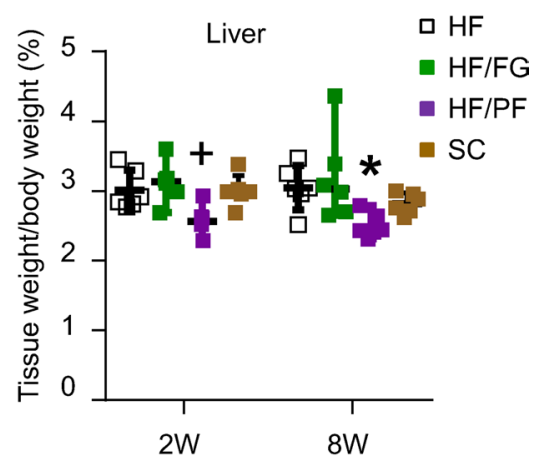

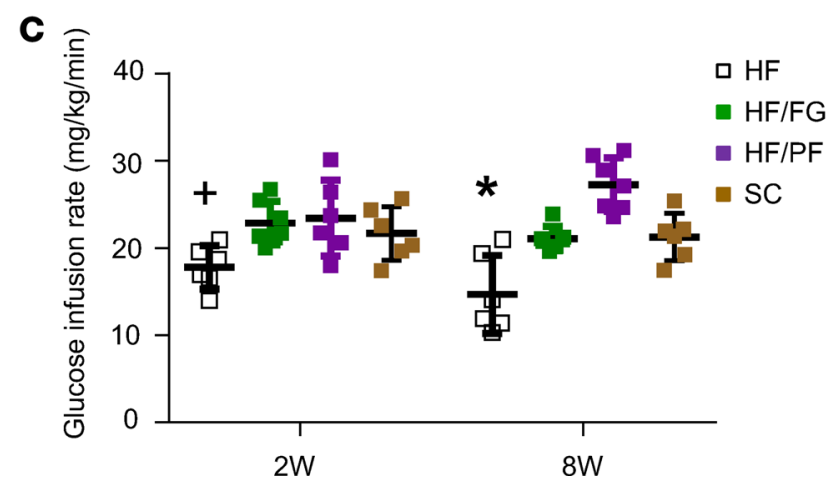

Figure 2. Fumagillin reduces white adipose tissue weights and improves insulin sensitivity in obese rats. Diet-induced obesity was induced in Wistar rats by feeding a high-fat (HF) diet for 12 weeks. A control group was fed a standard chow (SC) diet for the same time period. After the feeding period, rats were then subjected to dietary intervention periods of 2 or 8 weeks as described in Figure 1C. (A) Epididymal white adipose tissue (EWAT) weights for the groups described in Figure 1C, fed the indicated diets for 2 or 8 weeks ( $2 \mathrm{~W}, 8 \mathrm{~W}$, respectively). ${ }^{+} P<0.05$ compared with HF group after 2 weeks of diet intervention. ${ }^{\circledR} P<0.05$ when compared with HF and HF/FG groups after 2 weeks of diet intervention. ${ }^{\#} P<0.05$ when compared with the other 3 groups after 8 weeks of diet intervention. (B) Liver weights for the groups described in Figure $1 \mathrm{C}$, fed the indicated diets for 2 or 8 weeks ( $2 \mathrm{~W}$, $8 \mathrm{~W}$, respectively). ${ }^{+} P<0.05$ when compared with HF group after 2 weeks of diet intervention. ${ }^{*} P<0.05$ when compared with HF group after 8 weeks of diet intervention. In $\mathbf{A}$ and B, tissue weights were normalized to body weight. $n=6$ for each group. Data are presented as mean \pm SD. (C) Glucose infusion rate during hyperinsulinemic-euglycemic clamps. A cohort of animals treated as described in Figure 1 C were used. Data are presented as mean \pm SD for $n=5-7$ rats per group. ${ }^{+} P<$ 0.05 when compared with other groups after 2 weeks of diet intervention. ${ }^{*} P<0.05$ when compared with other groups after 8 weeks of diet interventions. For A-C, 2-tailed, unpaired $t$ tests were performed. $P<0.05$ with a Bonferroni correction was used to define statistical significance among groups.

In addition to showing the primary profiles for $\mathrm{VO}_{2}$, physical activity, $\mathrm{RER}$, and heat production, Figures 3 and 4 summarize each of those measurements as AUCs during the light cycle, the dark cycle, and the entire 24-hour observation period and provide the results of statistical tests of observed differences between the treatment groups. Remarkably, $\mathrm{VO}_{2}$ was significantly reduced during the light cycle, the dark cycle, and the full observation period, at both the 2-week and 8-week time points, in the HF/FG group compared with the HF/PF group (Figure 3A). Heat production was also significantly lower in the HF/FG group than in the HF/PF group at both time points (Figure 3B). Physical activity trended lower at 2 weeks and was significantly lower at 8 weeks in the HF/FG compared with HF/PF group (Figure 4A). RER was similar in the HF, HF/FG, and HF/PF groups in the light and dark cycles and over the full observation period, whereas, as expected, animals fed the grain-based SC diet had a higher RER than any of the HF groups at both the 2-week and 8-week time points (Figure 4B). Together, these data demonstrate that the dramatic and sustained weight loss in the HF/FG group compared with the HF/PF group is not explained by increased physical activity or energy expenditure. To the contrary, energy expenditure and physical activity were lower in HF/FG rats.

To investigate if the more relaxed feeding response of HF/FG rats was related to a taste aversion response to the fumagillin-supplemented food, we performed a diet preference test. When single-caged, SC-fed rats were given a mixture of equal portions of HF and HF/FG diets (the 2 diets are distinguishable by added food coloring), they began by eating approximately equal portions of each but then shifted to preferential consumption of the HF diet (Supplemental Figure 4D). This suggests that the HF/FG food 

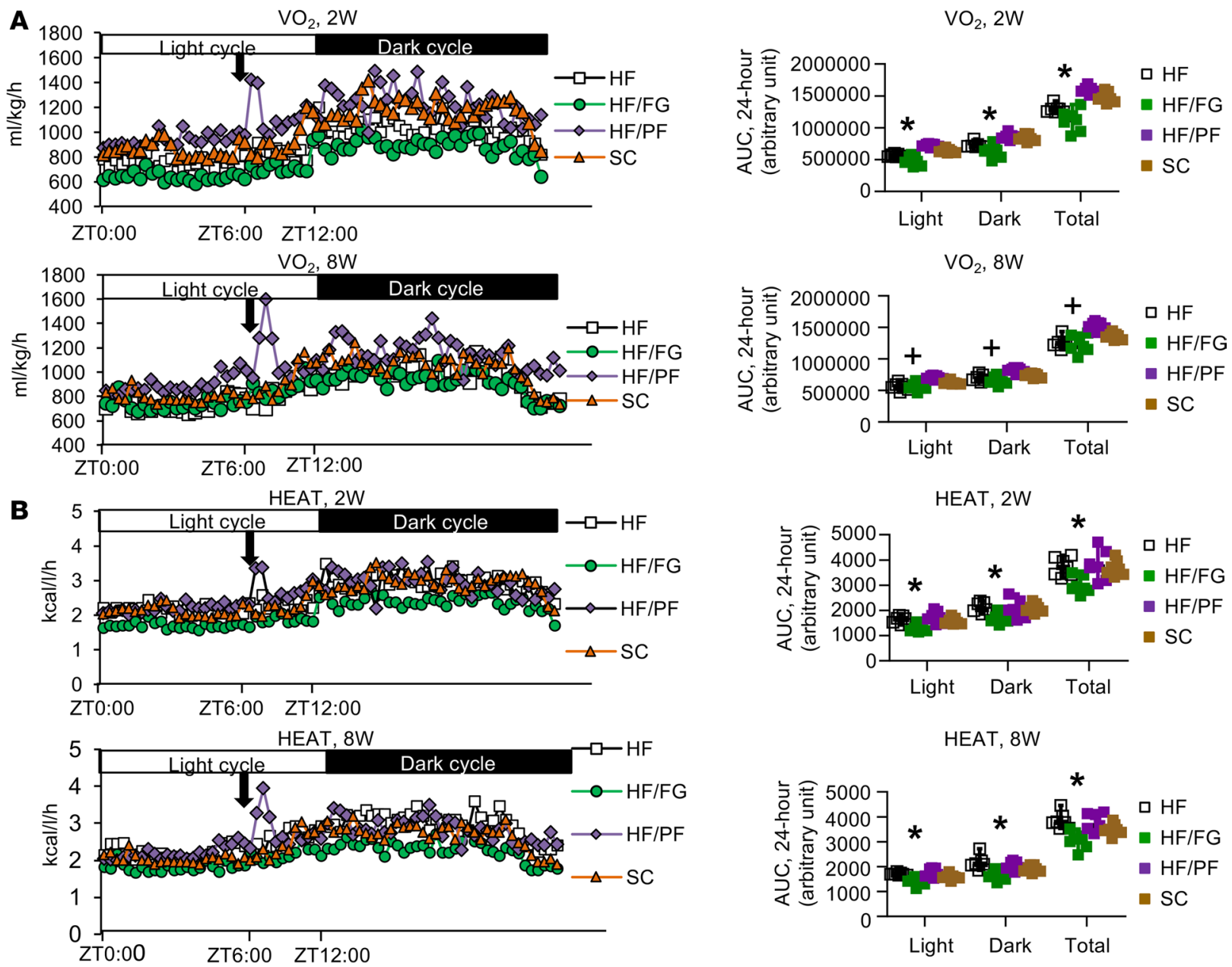

HEAT, 8W

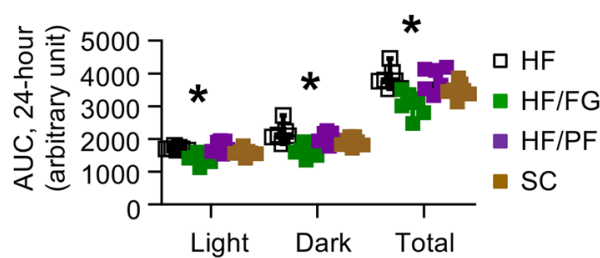

Figure 3. Fumagillin feeding affects oxygen exchange rate and heat production. The groups described in Figure $1 \mathrm{C}$ were studied in metabolic cages at 2 weeks or 8 weeks after the start of the dietary intervention period. (A) Oxygen exchange rate $\left(\mathrm{VO}_{2}\right.$ ) and (B) heat production (HEAT) are represented. For these measurements, upper panels represent data collected from the animals after 2 weeks and lower panels after 8 weeks of intervention. Left panels are raw data, and right panels summarize measurements as AUC. All groups received food once daily at ZT6:00 (1 pm), as shown by the downward-pointing arrows. Data for AUCs in the right panels are presented as mean \pm SD for $n=7$ for each group. ${ }^{*} P<0.05$ when compared with the other 3 groups; ${ }^{+} P<0.05$ when compared with $\mathrm{HF} / \mathrm{PF}$ group. Two-tailed, unpaired $t$ tests were performed. $P<0.05$ with a Bonferroni correction was used to define statistical significance among groups.

induces temporary taste aversion, possibly contributing to the lower food consumption in the early phases of the feeding study. However, this effect is not sustained, as the animals increase consumption of HF/ FG food, ultimately returning to near the rate of consumption exhibited by the HF group (Figure 1C). We also emphasize that this apparent diet preference for $\mathrm{HF}$ relative to HF/FG food is unlikely to explain the relaxed feeding behavior of HF/FG-fed rats. This is supported by a recent study in which injection of a different MetAP2 inhibitor, beloranib, caused consistent suppression of "frantic" eating and hyperphagia-related behaviors in a cohort of human subjects with Prader-Willi syndrome (10). Given that the drug was injected in those studies, the suppression of food-seeking behavior cannot be ascribed to direct taste aversion caused by oral ingestion of a MetAP2 inhibitor-containing diet.

Fumagillin treatment affects feeding rhythm and circadian gene expression. To further investigate the effects of fumagillin on feeding behavior, food intake was monitored multiple times daily after provision of food at ZT6:00 (1 pm). During the first 5 days of the experiment, food was provided ad libitum for both the $\mathrm{HF}$ and HF/FG groups. Once the rate of food intake was established for the HF/FG group over this time period, the HF/PF group and HF/FG groups were given an amount of food to closely model consumption of the HF/FG group ( $55 \%$ of the food consumed by animals in the ad libitum fed HF group). This amount was slightly more than actually consumed by the HF/FG group during ad libitum feeding $(52 \%$ of the HF group, average per day at these early-intervention time points). We reasoned that providing 
A
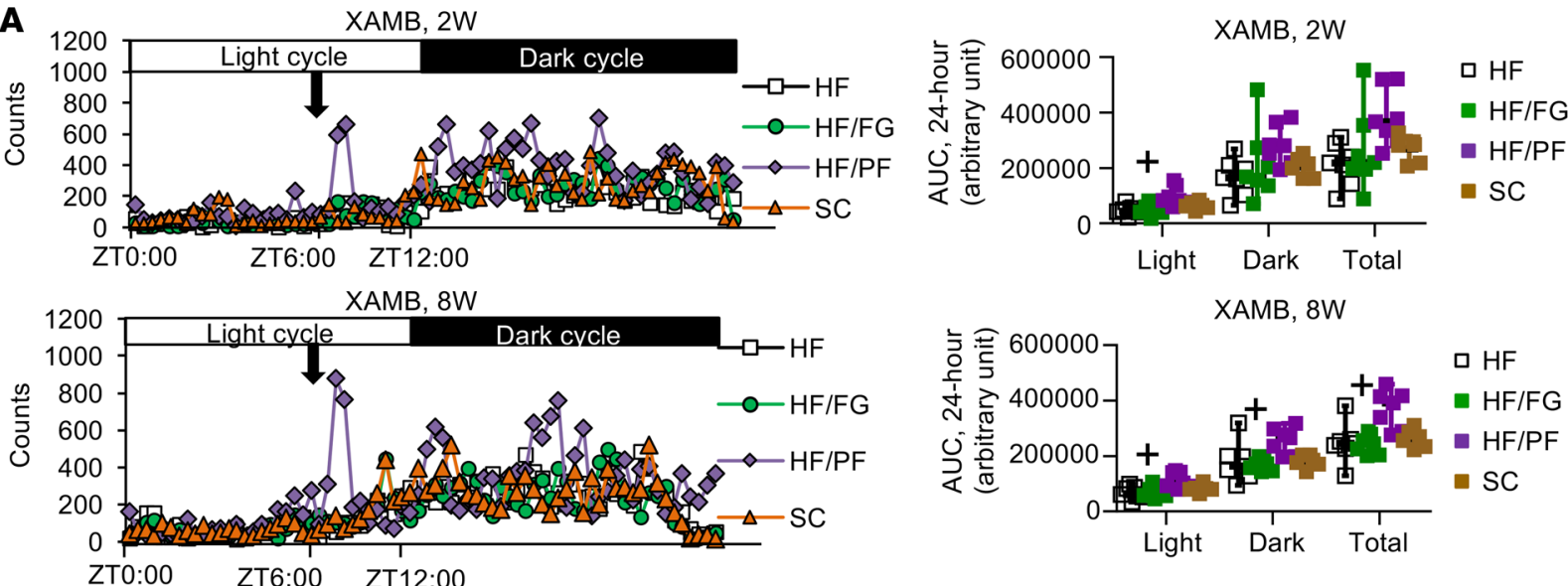

Light Dark Total

XAMB, 8W

B
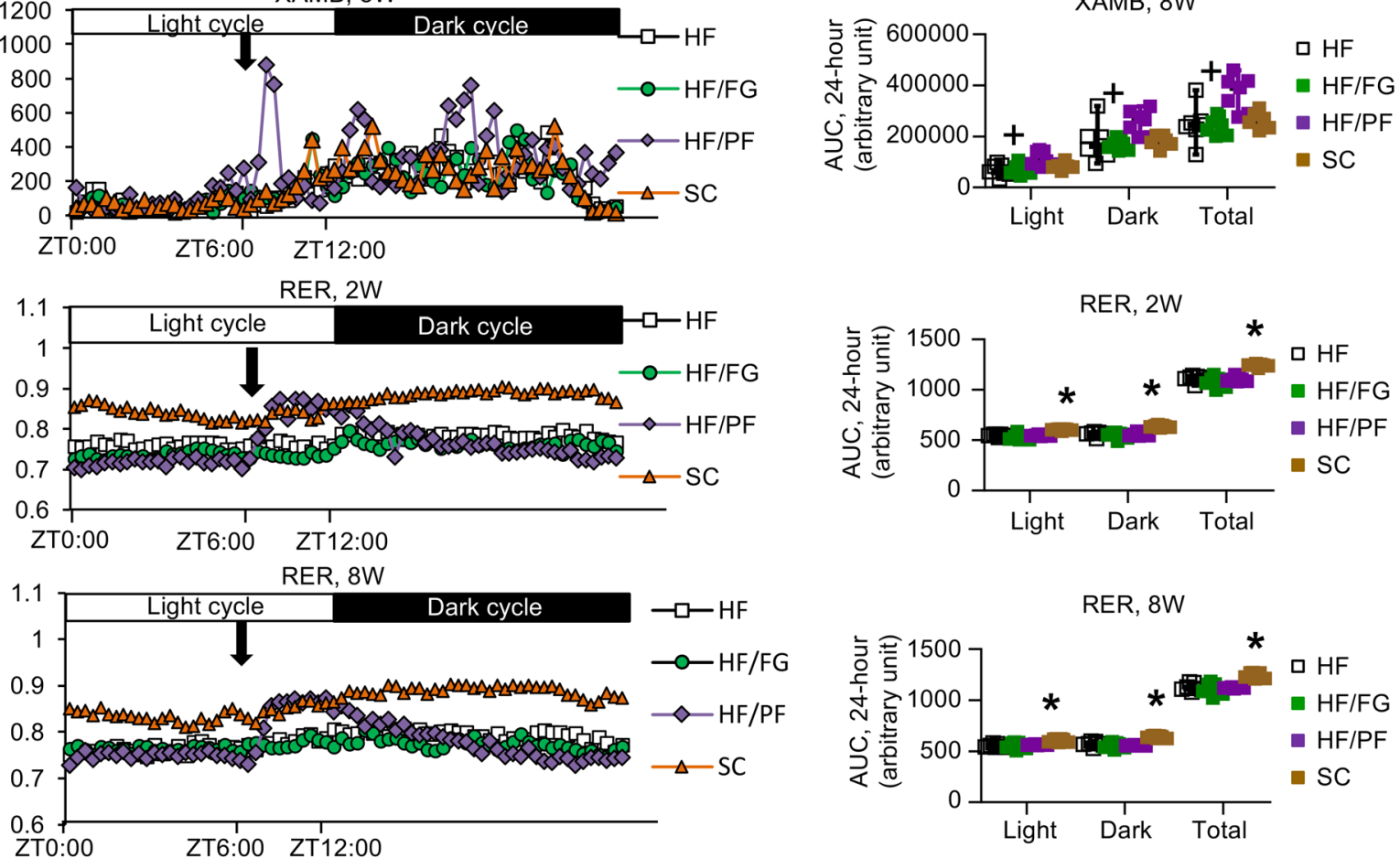

Figure 4. Fumagillin feeding affects ambulatory activity and has an acute effect on respiratory exchange rate (RER). The groups described in Figure $1 C$ were studied in metabolic cages at 2 weeks or 8 weeks after the start of the dietary intervention period. (A) Ambulatory activity (XAMB) and (B) respiratory exchange rate (RER) are represented. For these measurements, upper panels represent data collected from the animals after 2 weeks and lower panels after 8 weeks of intervention. Left panels are raw data, and right panels summarize measurements as AUC. All groups received food once daily at ZT6:00 $(1 \mathrm{pm})$, as shown by the downward-pointing arrows. Data for AUCs in the right panels are presented as mean \pm SD for $n=7$ for each group. ${ }^{*} P<0.05$ when compared with the other 3 groups; ${ }^{+} P<0.05$ when compared with HF/PF group. Two-tailed, unpaired $t$ tests were performed. $P<0.05$ with a Bonferroni correction was used to define statistical significance among groups.

the same food portions to the HF/PF or HF/FG groups would control for anxiety responses elicited by perception of limited food availability.

Animals in the ad libitum-fed HF group ate predominantly during the dark cycle from ZT14:00-2:00 (9 pm to $9 \mathrm{am}$ ) and consumed far less food in the light periods from ZT6:00-10:00 (1-5 pm) and ZT10:0014:00 (5-9 pm) $\left(P=5.7 \times 10^{-15} ;\right.$ Figure $\left.5 \mathrm{~A}\right)$. In the HF/FG group, food intake was reduced in all 3 time periods relative to the HF group $(P \leq 0.0024)$, although the general pattern of greater food consumption in the dark than in the light was retained (Figure 5B). Over the course of several days, animals in the HF/ $\mathrm{PF}$ group developed a distinct feeding pattern compared with the HF/FG or HF groups, with most food consumed in the first 8 hours in the light phase (Figure 5C). These data are consistent with the metabolic cage experiments summarized in Figures 3 and 4 that document increased energy expenditure and physical activity (mostly related to feeding) in the HF/PF group in the time period immediately after provision of food at ZT6:00 (1 pm).

These results suggest that fumagillin may serve to suppress aggressive feeding behavior under conditions of single-meal feeding. To determine if fumagillin also influenced genes involved in control of circadian rhythms related to food intake, we measured mRNAs encoding positive (Clock and Bmal1) and negative (PerI) regulators of the circadian clock in different sections of gastrointestinal tract and liver, as well as in WAT, all of which have been reported to contain a functional clock system (11-13). HF/PF rats had lower expression 

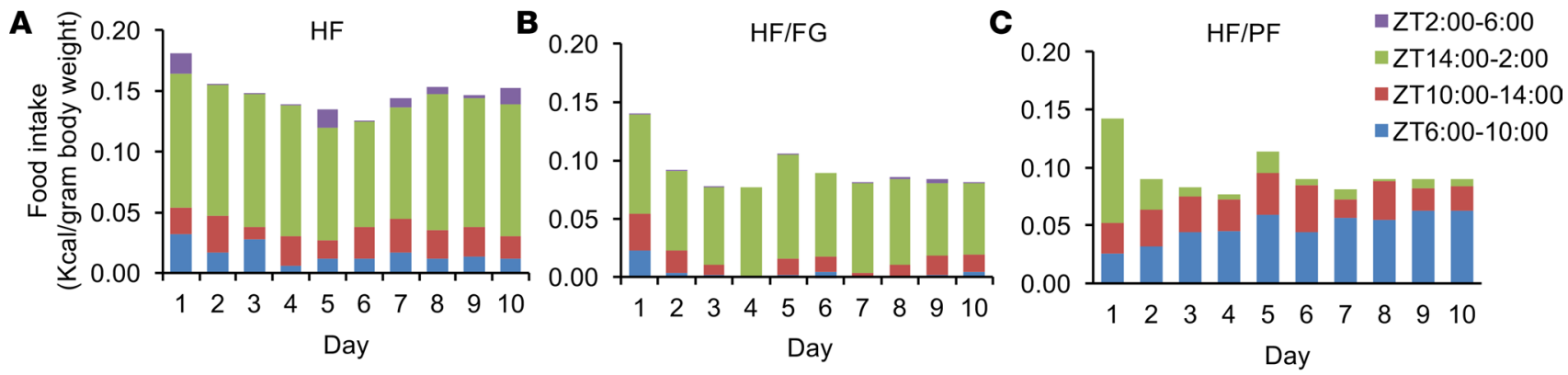

D
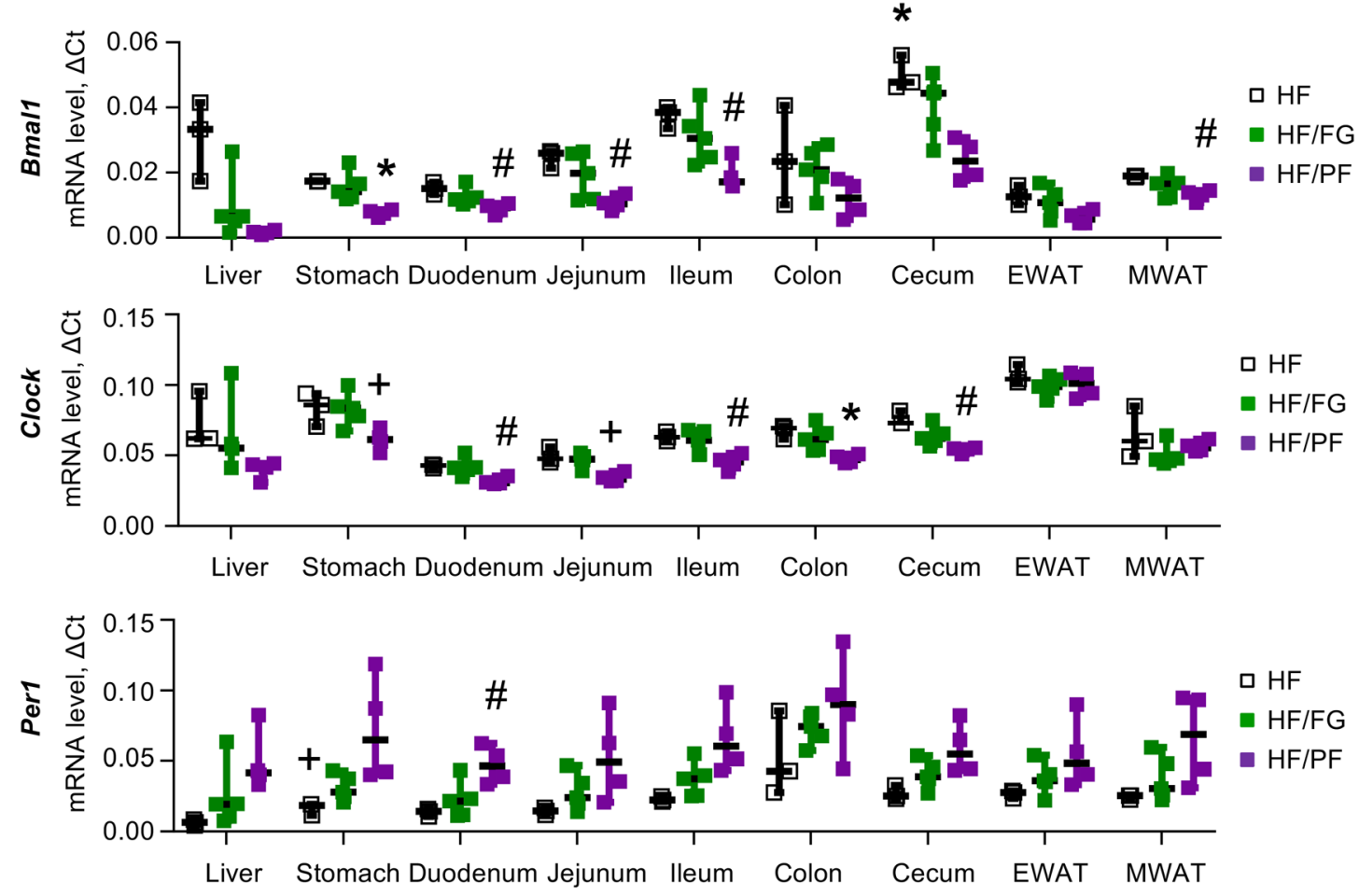

Figure 5. Fumagillin feeding affects feeding rhythm and expression of circadian genes. Wistar rats were fed a HF diet for 12 weeks and then subjected to an intervention period of 4 weeks, during which they consumed HF diet ad libitum (HF), HF diet with fumagillin (HF/FG), or an amount of HF food matched to the amount consumed by the HF/FG group (HF/PF). (A-C) Average food intake measured in these 3 groups of rats daily over 4 time periods after daily food provision at ZT6:00 (1:00 pm). The periods sampled were ZT6:00-10:00, ZT10:00-14:00, ZT14:00-2:00, and ZT2:00-6:00, equating to 1-5 pm, 5-9 pm, 9 pm-9 am, and 9 am-1 pm. $n=3,5$, and 4 for HF, HF/FG, and HF/PF groups, respectively. HF group compared with HF/FG or HF/PF groups for total food intake; $P<0.000009$. HF group compared with HF/FG group for food intake during ZT6:00-2:00 (1 pm-9 am); $P<0.04$. HF group compared with HF/PF group for food intake during all time periods except for ZT10:00-14:00 (5-9 pm); $P<0.04$. HF/FG group compared with HF/PF group for food intake during all time periods except for ZT2:00-6:00 (9 am-1 pm); $P<0.002$. (D) Expression levels of Bmal1 (upper panel), Clock (middle panel), and Per1 (lower panel) transcripts in liver, stomach, duodenum, jejunum, ileum, colon, cecum, epididymal white adipose tissue (EWAT), and mesenteric white adipose tissue (MWAT). Samples were collected after 10 days of diet intervention. Data represent means $\pm S D$ for $n=3,5$, and 4 for $H F, H F / F G$, and $H F / P F$ groups, respectively. ${ }^{*} P<0.05$ when compared with the other 2 groups. ${ }^{*} P<0.05$ when compared with the HF group. ${ }^{+} P<0.05$ when compared with $\mathrm{HF} / \mathrm{FC}$ group. For all panels, 2 -tailed, unpaired $t$ tests were performed. $P<0.05$ with a Bonferroni correction was used to define statistical significance among groups.

of Clock and Bmal1 and increased levels of Per1 mRNA in the stomach, colon, liver, and multiple regions of the small intestine compared with either the HF or HF/FG groups, whereas group differences were either not apparent or present as weak trends in epididymal or mesenteric WAT (Figure 5D). These findings suggest that fumagillin-induced alterations in feeding behavior and food intake may prevent entrainment of circadian genes in the liver and gastrointestinal tract that normally occur in response to caloric restriction.

Fumagillin induces energy conservation. To explore the effects of fumagillin on energy balance, we used another cohort of rats for analysis of body (rectal) temperatures. To simplify this study, we chose a single dietary intervention time point (4 weeks) intermediate between the 2- and 8-week time points shown in Figures 1-4. The weight loss profile of all rats studied after 4 weeks of intervention is shown in Supplemental Figures 2, A-D. HF/FG fed animals had significantly lower body temperatures compared with either HF/PF- or HF-fed 
A

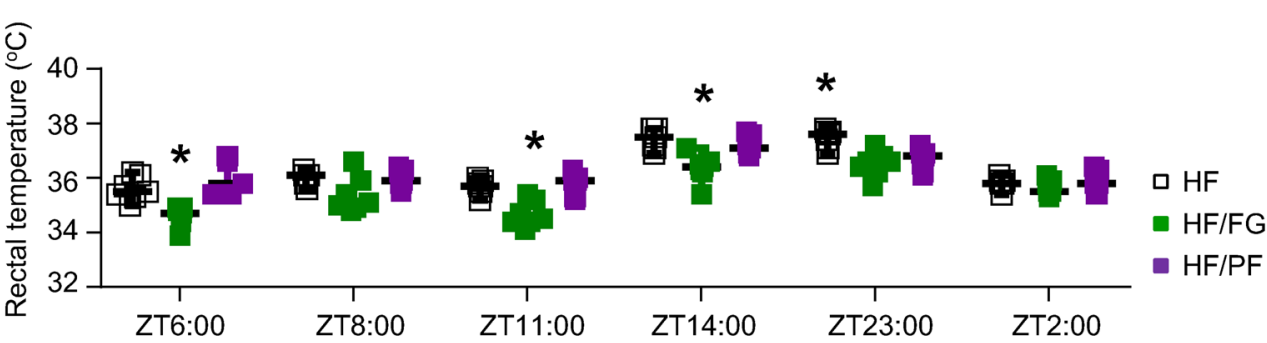

B
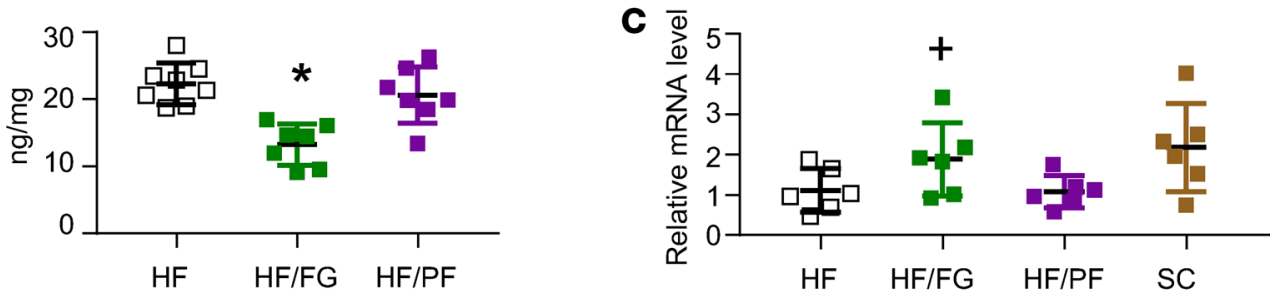

Catecholamines/CREA

FGF21

D

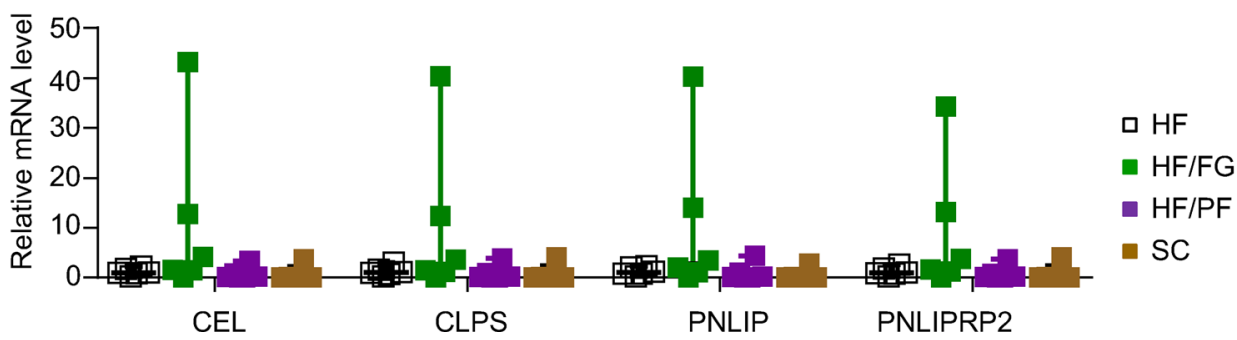

Figure 6. Fumagillin treatment affects body temperature, expression of torpor-related genes, and urine catecholamines. Rats were fed the HF diet for 12 weeks and then subjected to an intervention period of 2 or 4 weeks, during which time they consumed HF diet ad libitum (HF), HF diet with fumagillin (HF/ FG), or an amount of HF food matched to the amount consumed by the HF/FG group (HF/PF). (A) Following 4 weeks of diet intervention, rectal temperatures were measured in rats at 6 time points over a 20-hour period that covered light and dark cycles. Data are presented as mean $\pm S D$ for $n=7$ per group. ${ }^{*} P<0.05$ when compared with the other 2 groups. (B) Urine catecholamine levels, normalized to creatinine (CREA), in samples collected after 4 weeks of diet intervention. Data are presented as mean \pm SD for $n=8$ per group. ${ }^{*} P<0.05$ when compared with the other 2 groups. (C and D) Hepatic expression of torpor-related transcripts after 2 weeks of diet interventions. Data are presented as mean \pm SD. $n=6$ for each group. ${ }^{+} P=0.057$ when compared with $\mathrm{HF}$ group. Two-tailed, unpaired $t$ tests were performed. $P<0.05$ with a Bonferroni correction was used to define statistical significance among groups.

rats at both time points sampled in the dark cycle, and at 2 of 4 time points sampled in the light cycle $(n=7$ rats/treatment group; Figure 6A).

The reductions in physical activity and oxygen consumption (Figures 3 and 4), along with body temperature (Figure 6A), induced by fumagillin feeding could suggest a decrease in sympathetic tone. To test this, we collected urine samples and measured catecholamine levels. HF/FG feeding led to a significant reduction of urine catecholamine levels relative to either the HF or HF/PF group (Figure 6B), consistent with a suppression of sympathetic tone.

The chronic lowering of body temperature in the HF/FG group suggests that fumagillin may induce a condition resembling torpor, as experienced in hibernating animals, although it should be emphasized that physical activity of the HF/FG group, while clearly reduced compared with the HF/PF group, was similar to that of the HF group (Figures 3 and 4). To investigate this notion further, we measured expression of torpor-related genes in the liver $(14,15)$. Two weeks after intervention, several genes considered as molecular markers of torpor, including fibroblast growth factor 21 (FGF21), carboxyl ester lipase ( $\mathrm{Cel}$ ), pancreatic colipase (Clps), pancreatic lipase (Pnlip), and pancreatic lipase-related protein 2 (Pniprp2), trended to be expressed at higher levels in the livers of animals in the HF/FG group compared with either the HF or HF/PF group (Figures 6, C and D). These changes did not achieve statistical significance due to the varying responses among individual HF/FG animals and to the fact that the transcripts were undetectable in livers of several rats in the HF/PF control group, similar to what has been reported for induction of torpor genes by FGF21 (14). These results demonstrate that HF/FG feeding induces a 
chronic change in energy balance that is not explainable simply by a reduction of food intake, since these changes were not observed in $\mathrm{HF} / \mathrm{PF}$ animals.

Fumagillin treatment influences gastrointestinal energy harvest and secretion of gastrointestinal hormones, but not gastric emptying. Results summarized so far leave us with the question of how HF/FG feeding causes more extensive and sustained weight loss compared with HF/PF feeding, despite the effect of the former intervention to lower energy expenditure and physical activity. One possibility is that fumagillin could alter gut function and energy harvest. Consistent with this idea, we found - using bomb calorimetry - that HF/ FG rats retained significantly more energy in their feces than either the HF or HF/PF groups (Supplemental Figure 5A), suggesting that fumagillin alters energy extraction/absorption in the gut.

We also performed fasting/refeeding experiments in HF/FG- and HF/PF-fed animals and measured the mass of residual, dried stomach contents 72 hours after the conclusion of the meal (16). At that time point, members of the HF/FG group had significantly more residual food in the gut compared with the HF/PF group (Supplemental Figure 5B). However, it should be noted that it took approximately 1.5 hours for animals in the HF/FG group to finish eating all of the food provided, compared with only 10 minutes for animals in the HF/PF group. In a separate experiment, we gave liquid acetaminophen via oral gavage, measured the rate of appearance of acetaminophen in the serum, and observed no significant differences between the 2 groups (Supplemental Figure 5C). In aggregate, these findings suggest no intrinsic difference in gastric emptying or absorptive function; rather, they suggest that the greater retention of food in the stomach of HF/FG rats is related to their slower rate of food consumption rather than a change in gastric-emptying rate.

Finally, since reductions of caloric intake and body weight occurred within 2 days of initiation of HF/ FG feeding, we investigated the possible involvement of gastrointestinal hormones known to affect food intake and energy balance. Blood samples were collected 2 days after the start of interventions, at ZT6:00 (1 pm) for the HF and HF/FG groups and at ZT0:00 (7 am) for the HF/PF group. The HF/FG group had significantly higher levels of GLP-1 than the HF/PF group (Supplemental Figure 6A); GLP-1 is thought to work centrally to suppress food intake. In addition, compared with the HF group, HF/PF animals exhibited a clear increase in circulating levels of ghrelin, a hormone that promotes food intake, but this increase was not observed in HF/FG rats (Supplemental Figure 6B). The ghrelin/GLP-1 ratio was significantly higher in HF/PF compared with HF rats (Supplemental Figure 6C). Levels of adiponectin were also significantly higher in HF/FG compared with HF and HP/PF animals (Supplemental Figure 6D). Together, these data are consistent with a potential role of GLP-1 in mediating the decrease in food intake in HF/ FG rats. This, coupled with the lack of increase in ghrelin in HF/FG animals despite their caloric restriction, could contribute to the more relaxed feeding behavior of animals in the HF/FG group. Short-chain fatty acids (SCFA) produced by the microbiota have been linked previously to gut hormone secretion (17, 18). We found that levels of acetate in cecal contents harvested from HF/FG rats were significantly higher than in either the HF or HF/PF groups; propionate and butyrate levels followed the same trend, but the differences did not achieve statistical significance (Supplemental Figure 6E). SCFA are thought to bind to GPCRs GPR41 and GPR43 in L cells of the small intestine to stimulate GLP-1 release (19).

Metabolomics analyses. Metabolomics analyses revealed a wide array of differences between HF/FG and $\mathrm{HF} / \mathrm{PF}$ groups, which are likely due to the differences in feeding behavior (Supplemental Table 2). For all animals, food was provided once daily at ZT6:00 (1 pm). Based on our finding of clear differences in feeding behavior between HF/FG and HF/PF animals (Figures 3 and 4), this meant that the HF/PF group was eating all its food in a rapid fashion and then experiencing a long fasting period prior to sacrifice the next day at ZT6:00 (1 pm). In contrast, the HF/FG group was nibbling food slowly throughout the night, meaning that these animals were less acutely fasted at the time of sacrifice. As a result, the HF/PF group had large increases in fatty acid-derived acylcarnitines and ketone metabolites compared with the HF or HF/FG groups, consistent with activation of lipolysis and oxidation of fatty acids, a hallmark of the fasted state (Supplemental Table 2). Moreover, analysis of urine samples indicated significant reduction of urea nitrogen and urea levels (normalized to creatinine [CREA] levels) in the HF/FG compared with HF/PF and HF groups (Supplemental Figure 7, A and B), as well as increases in levels of multiple urinary amino acids (Supplemental Figure 7C); these results are suggestive of reduced rates of amino acid oxidation in HF/FG rats and possible caloric spilling that could contribute to the more sustained weight loss in the HF/FG group.

Two other metabolic differences between the HF/FG and HF/PF groups were particularly noteworthy. First, we found that HF/FG-treated rats had levels of glycogen in liver and skeletal muscle that were 
A

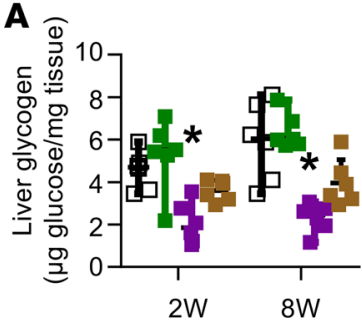

B

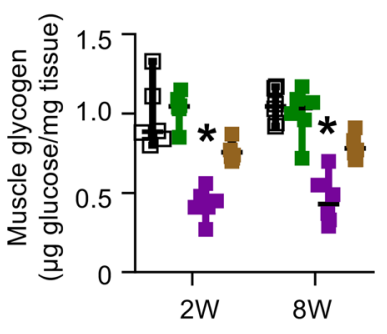

C

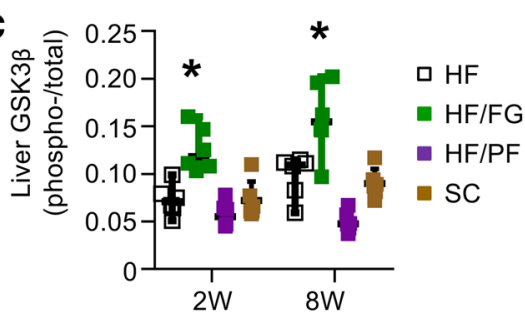

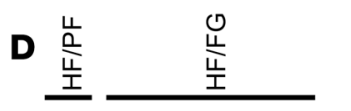

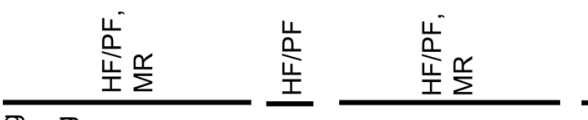
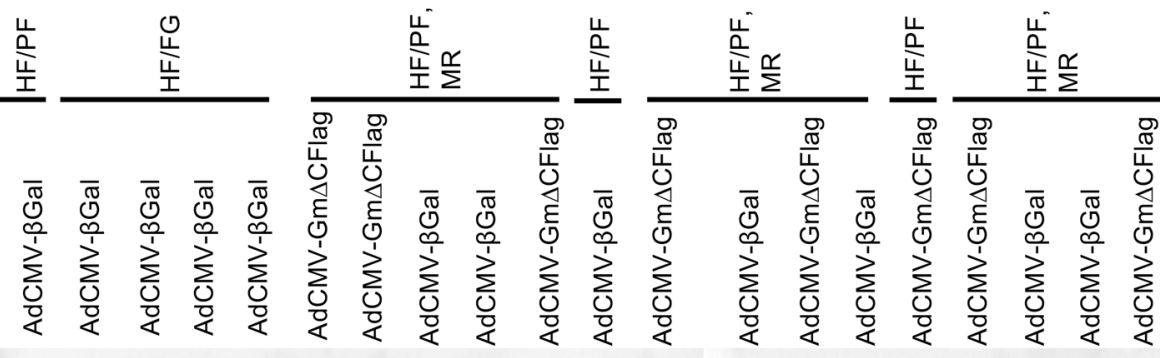

-
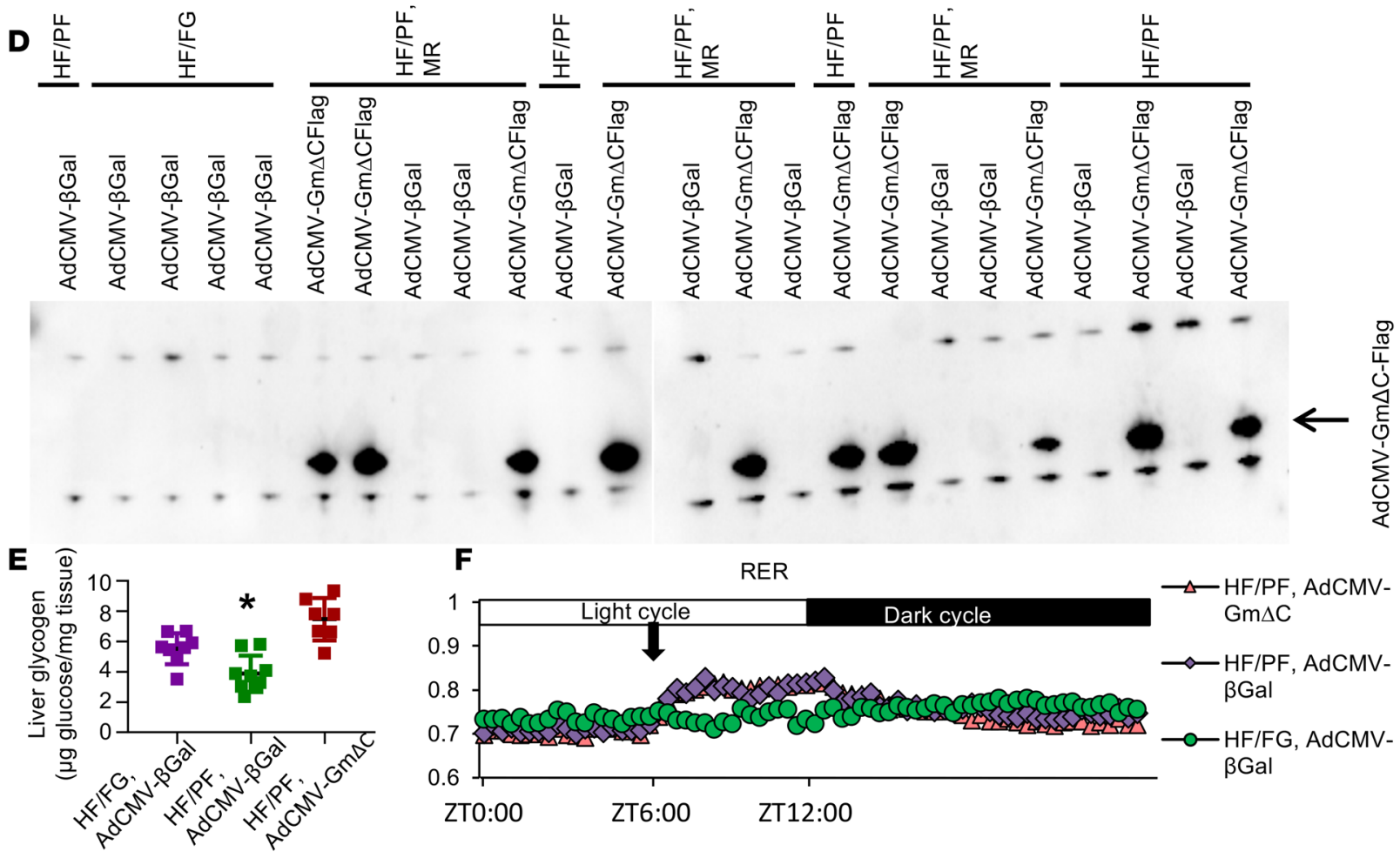

Figure 7. Fumagillin treatment affects glycogen levels, but hepatic glycogen is not the mediator of fumagillin-induced changes in feeding behavior. First 3 panels: rats were fed HF or SC diet for 12 weeks and then subjected to dietary intervention periods of 2 or 8 weeks, as described in Figure $1 C$. Liver and muscle samples were collected for measurement of glycogen levels or phospho-GSK3 $\beta$ levels at ZT6:00 (1 pm) at the 2-week or 8-week time points. Last 3 panels: rats were fed on HF diet for 12 weeks and then subjected to an intervention period of 4 weeks, during which rats were fed HF diet with fumagillin ad libitum (HF/FG) or an amount of HF food matched to the amount consumed by the HF/FG group (HF/PF), in the presence or absence of methionine restriction (MR). Liver samples were collected at ZT6:00 (1 pm), immediately prior to the normal once-daily provision of food. (A) Hepatic glycogen levels. Data are mean \pm SD for $n=6$ for each group. ${ }^{*} P<0.05$ when compared with other groups at each time point. (B) Glycogen levels in gastrocnemius muscle. Data are mean $\pm S D$ for $n=6$ for each group. ${ }^{*} P<0.05$ when compared with other groups at each time point. (C) Ratio of phosphorylated GSK3 $\beta$ compared with total GSK3 $\beta$ in liver. Data are mean \pm SD for $n=6$ for each group. ${ }^{*} P<0.05$ when compared with other groups at each time point. (D) Immunoblot analysis of liver samples obtained from rats treated with adenoviruses expressing either a Flag-tagged C-terminal deleted version of the muscle isoform of glycogen-targeting subunit of protein phosphatase 1 ( $\mathrm{Gm} \Delta \mathrm{C}-\mathrm{Flag}$ ) (21) or $\beta$-galactosidase ( $\beta$ Gal) and continuously fed the indicated diets for 1 week. (E) Hepatic glycogen levels in rats treated with the indicated adenoviruses. Data are mean \pm SD for $n=5-7$ animals per group. ${ }^{*} P<0.02$ when compared with the other groups. (F) Changes in RER in response to daily provision of food at ZT6:00 (1 pm) (downward arrow) in rats fed the indicated diets and treated with the indicated adenoviruses. All diets in this experiment contained normal methionine levels. $n=5-7$ for each treatment group. For all panels, 2-tailed, unpaired $t$ tests were performed. $P<0.05$ with a Bonferroni correction was used to define statistical significance among groups.

similar to animals fed on HF diet ad libitum, whereas liver and muscle glycogen levels were significantly lower in the HF/PF group (Figure 7, A and B). These findings may help to explain the selective decrease in liver weight noted in the HF/PF compared with the $\mathrm{HF}$ and $\mathrm{HF} / \mathrm{FG}$ treatment groups (Figure 2B). Consistent with these findings, levels of phosphorylated glycogen synthase kinase $3 \beta$ (GSK3 $\beta$ ) were significantly increased in the livers of HF/FG compared with HF/PF rats at both the 2- and 8-week time points of the intervention (Figure 7C) and in skeletal muscle at 8 weeks (data not shown); phosphorylation of GSK $3 \beta$ inhibits its activity and leads to less inhibitory phosphorylation of glycogen synthase, contributing to more active glycogen synthesis. Second, animals that ate the HF/FG diet had significantly 
lower liver methionine levels than either the HF or HF/PF group (Supplemental Table 2). This observation suggests that MetAP2, the fumagillin target enzyme that cleaves $\mathrm{N}$-terminal methionine residues from nascent proteins, is a significant regulator of steady state methionine levels.

Modulation of hepatic glycogen and/or dietary methionine levels does not affect feeding behavior of pair-fed animals. Prior studies have implicated liver glycogen (19) and dietary methionine (20) as modulators of feeding behavior in rodents. This led us to test the hypothesis that the reduced hepatic glycogen levels and/or elevated methionine levels in HF/PF rats contribute to their sense of starvation and abrupt feeding behaviors. To test this idea, we used recombinant adenovirus to overexpress a modified version of the muscle isoform of the glycogen-targeting subunit of protein phosphatase-1, known as $\mathrm{Gm} \Delta \mathrm{C}$-Flag. We have previously demonstrated that adenovirus-mediated expression of this construct in the livers of streptozotocin-treated diabetic rats raises hepatic glycogen levels, lowers blood glucose levels, and attenuates hyperphagia (21). The AdC$\mathrm{MV}-\mathrm{Gm} \Delta \mathrm{C}$-Flag adenovirus was injected into $\mathrm{HF} / \mathrm{PF}$ rats 4 weeks after initiation of their feeding protocol, and their behaviors in metabolic cages were compared with those of HF/PF or HF/FG rats injected with a control (AdCMV- $\beta$-galactosidase; AdCMV- $\beta$ Gal adenovirus ( $n=5-7$ animals/treatment group). Injection of AdCMV-Gm $\Delta \mathrm{C}$-Flag into $\mathrm{HF} / \mathrm{PF}$ rats increased expression of $\mathrm{Gm} \Delta \mathrm{C}$, as expected (Figure 7D), and raised liver glycogen to the levels found in HF/FG rats; levels in both groups were significantly greater than those observed in HF/PF rats injected with the control AdCMV- $\beta$ Gal vector (Figures $7 \mathrm{E}$ ). However, "clamping" liver glycogen at elevated levels in $\mathrm{HF} / \mathrm{PF}$ rats did not change feeding behavior. Like members of the HF/PF/AdCMV- $\beta$ Gal control group, these animals exhibited abrupt increases in ambulatory activity, $\mathrm{VO}_{2}$, and RER in response to provision of food (representative of all of these responses; only the changes in RER are shown in Figure 7F).

We also tested the potential role of reduced hepatic methionine levels on feeding. To do this, we created a HF, methionine-restricted (HF/MR) diet in an attempt to mimic the effects of fumagillin on lowering of methionine levels (Supplemental Table 1). We pair-fed the HF/MR diet to match the rate of HF/FG food intake (HF/PF, MR). HF/MR feeding was performed in rats treated with AdCMV-Gm $\triangle \mathrm{C}$-Flag or AdC$\mathrm{MV}-\beta \mathrm{Gal}$ (for levels of hepatic expression of $\mathrm{Gm} \Delta \mathrm{C}$ achieved with the recombinant virus, see Figure $7 \mathrm{D}$; for liver glycogen levels, see Supplemental Figure 8A). There were no statistically significant differences in caloric intake (kcal/g body weight) or body weight among the 3 study groups (HF/FG + AdCMV- $\beta$ Gal; HF/ PF, MR + AdCMV- $\beta$ Gal; HF/PF, MR + AdCMV-Gm $\Delta$ C-Flag; data not shown). HF/PF, MR-fed animals exhibited a trend for lowering of hepatic methionine levels compared with HF/FG animals (Supplemental Figure 8B), but methionine restriction did not significantly suppress the burst in RER or heat production in response to $\mathrm{HF} / \mathrm{PF}$ feeding, regardless of whether liver glycogen levels were maintained at high levels (data for RER shown in Supplemental Figure 8C; $n=5-7$ animals evaluated for each parameter/treatment group). Thus, we conclude that fumagillin-mediated increases in hepatic glycogen content or decreases in methionine levels do not explain differences in feeding behavior of HF/FG compared with HF/PF rats.

The gut microbiota is implicated in fumagillin-mediated expression of genes involved in energy conservation but not appetite suppression. The observed changes in energy extraction, cecal SCFA concentrations, and gut hormone levels suggest a testable hypothesis - namely, that fumagillin may exert effects on the host, in part, by altering the functional properties of the gut microbiota. 16S rRNA gene sequencing of fecal samples of $\mathrm{HF} / \mathrm{FG}$ and $\mathrm{HF} / \mathrm{PF}$ rats did not reveal significant differences between the composition of their gut microbiota. However, this metric does not reflect changes in composition at the strain-level or changes in gene expression that could be induced by fumagillin. Therefore, we performed microbiota transplant experiments to directly test whether the microbiota was causally related to the physiologic and metabolic phenotypes produced by fumagillin. Male, 10- to 12-week-old, germfree C57BL/6J mice were switched to the HF diet for 2 weeks (run-in period). Two groups of 5 germfree mice each were colonized with cecal microbiota harvested from a HF/FG rat, while 2 groups of 5 germ-free mice each received transplanted cecal microbiota from a HF/PF rat. The selected microbiota donors were representative of their treatment groups, as judged by their body weight and feeding phenotypes, as well as by the results of a phylogenetic dissimilarity metric applied to bacterial $16 \mathrm{~S}$ rRNA datasets generated from their cecal microbial communities (Supplemental Figure 9A). The first group of gnotobiotic mice colonized with $\mathrm{HF} / \mathrm{FG}$ or $\mathrm{HF} / \mathrm{PF}$ rat donor microbiota were fed the $\mathrm{HF}$ diet ad libitum, and food intake was measured over 3 time periods (ZT2:00-6:00, ZT6:00-14:00, and ZT14:00-2:00, equivalent to 9 am-1 pm, 1 pm-9 pm, and 9 pm-9 am, respectively). No significant differences in total food intake or food intake as a function of time period were observed in any of 
the groups of recipient mice (Figure 8, A and B). In a second set of experiments, germ-free mice colonized with cecal microbiota from HF/FG or HF/PF rats were fed ad libitum for 6 days and were then subjected to a $10 \%$ reduction in food portion relative to the amount consumed during the ad libitum feeding period. Caloric restriction did not elicit significant differences in the temporal pattern of feeding or total food intake in animals harboring microbiota from $\mathrm{HF} / \mathrm{FG}$ versus $\mathrm{HF} / \mathrm{PF}$ mice (Figure 8, C and D). Specifically, caloric restriction of the 2 groups of mice caused a similar increase in aggressive feeding behavior (occurring in the ZT6:00-10:00 [1-5 pm] period immediately after food provision) in the 2 groups of animals. In both experiments, there was no difference in body weight between the 2 groups of mice that received either HF/FG or HF/PF rat donor microbiota (Supplemental Figure 10).

We subsequently characterized torpor-related and circadian gene expression in livers of the mice studied in Figure 8, A-D. We found that animals containing microbiota from HF/FG rats exhibited a strong trend for induction of torpor marker genes such as Cel, Clps, and Pnlip, in both the ad libitum-fed and calorically restricted states, relative to mice harboring microbiota from $\mathrm{HF} / \mathrm{PF}$ rats (Figure $8 \mathrm{E}$ ). In contrast, no differences were observed in hepatic expression of the circadian genes Bmall and Clock in mice harboring microbiota from $\mathrm{HF} / \mathrm{FG}$ versus $\mathrm{HF} / \mathrm{PF}$ rats (Figure $8 \mathrm{~F}$ ). These findings suggest that the aggressive pattern of feeding behavior induced by caloric restriction, as well as the resulting changes in circadian gene expression, are regulated independent of the microbiota, whereas the gut community appears to have a role in regulating expression of torpor genes, perhaps relating to the energy preservation phenotype observed in response to fumagillin-induced caloric restriction.

To understand whether the abundance of certain gut bacterial species was correlated with these transmissible phenotypes, we performed indicator species analyses $(22,23)$ using $16 \mathrm{~S}$ rRNA gene sequencing datasets generated from fecal samples collected 2, 5, and 11 days after gavage of the rat donor gut microbial community, as well as cecal samples obtained at the time of sacrifice on day 11. Indicator species analysis identifies organisms associated with particular habitat types using the indicator species value (23). Using this approach, we identified 97\%ID OTUs (Operational Taxonomic Units sharing $\geq 97 \%$ nucleotide sequence identity) as associated with fumagillin-treated donor microbiota if they (i) were significantly more likely to be present in samples taken from mice colonized with this rat donor's microbiota and/or (ii) had significantly greater relative abundance in recipient mice harboring this community. We found eleven 97\%ID OTUs (across fecal samples and cecal samples taken at sacrifice) that distinguish the groups of animals that exhibited divergent torpor gene expression profiles (Supplemental Figure 9 and Supplemental Table 3). Additional indicator species analyses performed on the cecal microbiota of the treatment groups from which the donors were selected yielded 1 bacterial OTU among the 11 (OTU no. 296726, unclassified phylogeny) that was indicative of fumagillin treatment $(P<0.005$, uncorrected; Supplemental Figure 9B).

Finally, to test whether fumagillin has effects on energy balance independent of the gut microbiota, we fed another cohort of uncolonized, germ-free mice with HF or HF/FG diets. A significant decrease in body weight was observed in animals fed the HF/FG diet compared with mice receiving the HF diet with no fumagillin added. The difference was already significant within 2 days of administration of the diets, and it remained significant through 9 days of feeding (Figure 9). We then swapped the diets so that the group fed the HF/FG diet for the first 9 days was now eating HF diet without fumagillin, and the group eating the HF diet was now consuming the HF/FG diet. Rapid weight loss was observed in the group switched to the HF/FG diet, whereas weight regain was observed in the group switched to the HF diet. In aggregate, the studies shown in Figures 8 and 9 support the conclusion that fumagillin confers its effects on feeding behavior by mechanisms that are independent of the gut microbiota.

\section{Discussion}

In the current study, we have used a model of DIO in rats to study the effects of the natural product MetAP2 inhibitor fumagillin on weight loss and energy balance. We confirmed the findings of a previous study in DIO mice (7) in DIO rats by showing that fumagillin causes a larger amount of weight loss than pair feeding. Moreover, fumagillin feeding maintained weight loss over a 30-day period in DIO mice (7) and out to 8 weeks in the current DIO rat study. In contrast, the body weight of pair-fed animals in both studies began to rise over the course of the intervention period and were on a trajectory to return to baseline levels. We demonstrate that fumagillin induces sustained weight loss by mechanisms that reach beyond those activated by simple caloric restriction to include a remarkable set of physiologic, behavioral, hormonal, and biochemical changes. 
A
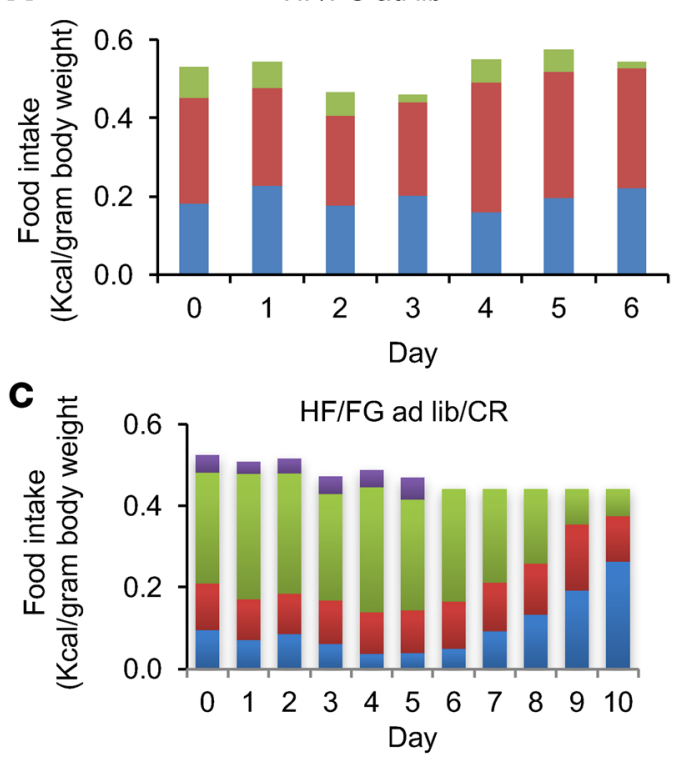

B

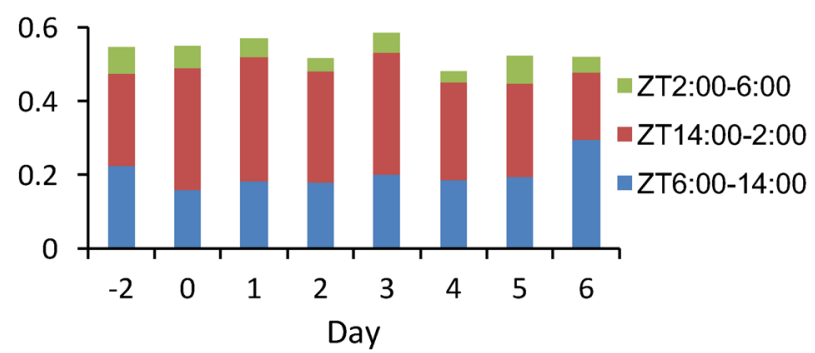

D

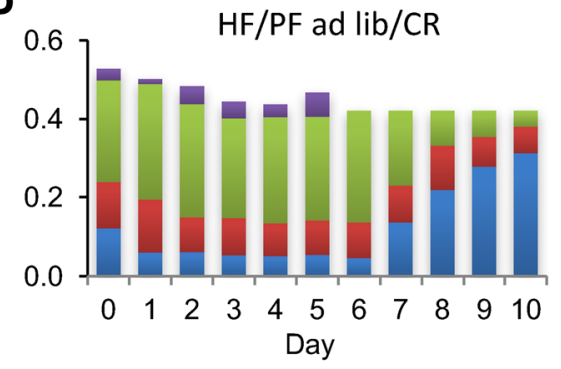

- ZT2:00-6:00

«TT14:00-2:00

- ZT 10:00-14:00

=ZT6:00-10:00

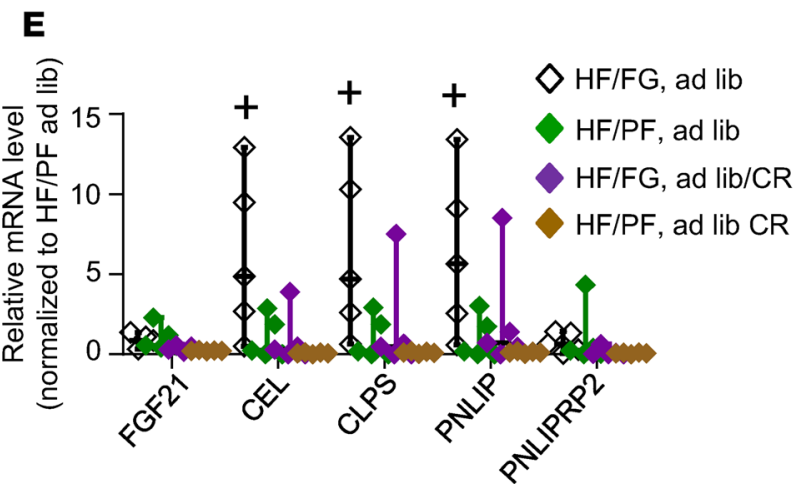

$\mathbf{F}$

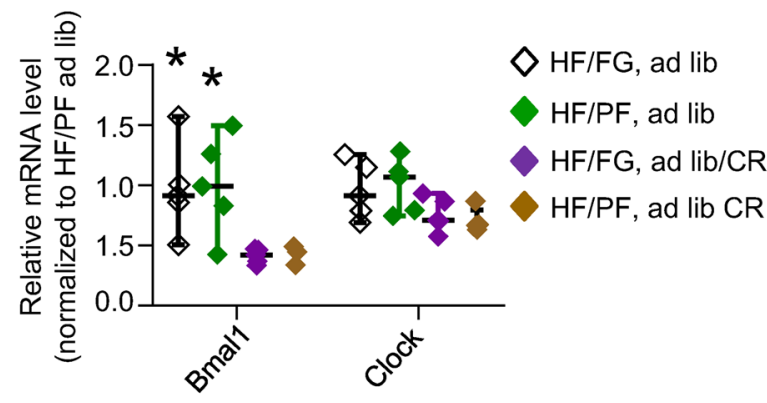

Figure 8. Feeding rhythms and hepatic torpor and Clock gene expression of gnotobiotic mice receiving cecal microbiota transplants from HF/FG or HF/ PF rats. All of the experiments in A-F, are from $n=5$ mice/group. (A) Daily accumulated food intake of mice colonized with the cecal microbiota of a HF/FC rat. HF diet was provided ad libitum. (B) Daily accumulated food intake of mice colonized with the cecal microbiota of a HF/PF rat. HF diet was provided ad libitum. (C) Daily accumulated food intake of mice colonized with the cecal microbiota from a HF/FG rat. HF diet was provided ad libitum from experimental day $1-5$ and then restricted by $10 \%$ from day $6-10$. (D) Daily accumulated food intake of mice that were the recipients of a cecal microbiota transplant from a HF/PF rat. HF diet was provided ad libitum from day $1-5$ and then restricted by $10 \%$ from day $6-10$. (E) Expression of hepatic torpor genes in mice from the studies shown in $\mathbf{A}-\mathbf{D} .{ }^{+} P=0.06$ when compared with HF/FG ad libitum/CR group. (F) Expression of hepatic circadian genes in mice from the studies shown in $\mathbf{A}-\mathbf{D}$. ${ }^{*} P<0.05$ when compared with the corresponding calorically restricted groups. In $\mathbf{E}$ and $\mathbf{F}$, data are presented as mean $\pm \mathrm{SD}$, and 2-tailed, unpaired $t$ tests were performed. $P<0.05$ with a Bonferroni correction was used to define statistical significance among groups.

The most striking finding of the current study was a clear effect of fumagillin on feeding behavior. Whereas HF/PF rats consumed food rapidly and aggressively upon presentation in our once-daily feeding paradigm, HF/FG rats ate much more slowly and ultimately consumed less food than rats fed the HF diet ad libitum. This nonchalant feeding behavior of HF/FG rats was linked to differences in expression of key circadian genes in multiple visceral organs compared with HF/PF rats (Figure 5D). These findings suggest that fumagillin-induced alterations in feeding behavior and food intake may prevent entrainment of circadian genes in the liver and gastrointestinal tract in response to caloric restriction. We note that the aggressive feeding response observed in HF/PF rats occurred when they were fed daily at ZT6:00 (1 pm), which is in the middle of the light cycle, when feeding is suppressed in HF- or HF/FG-fed rats. We became concerned that the perception of a limited portion size in HF/PF rats may have caused anxiety, contributing to the aggressive response. However, when portion size was reduced to a similar extent in HF/FG rats, aggressive feeding was not activated. Thus, if perception of portion size is a factor in aggressive feeding of HF/PF rats, fumagillin prevents this response. Consistent with this reduction in aggressive food-seeking behavior in rats, treatment with the fumagillin analog beloranib was found to strongly reduce severe hyperphagia-related 


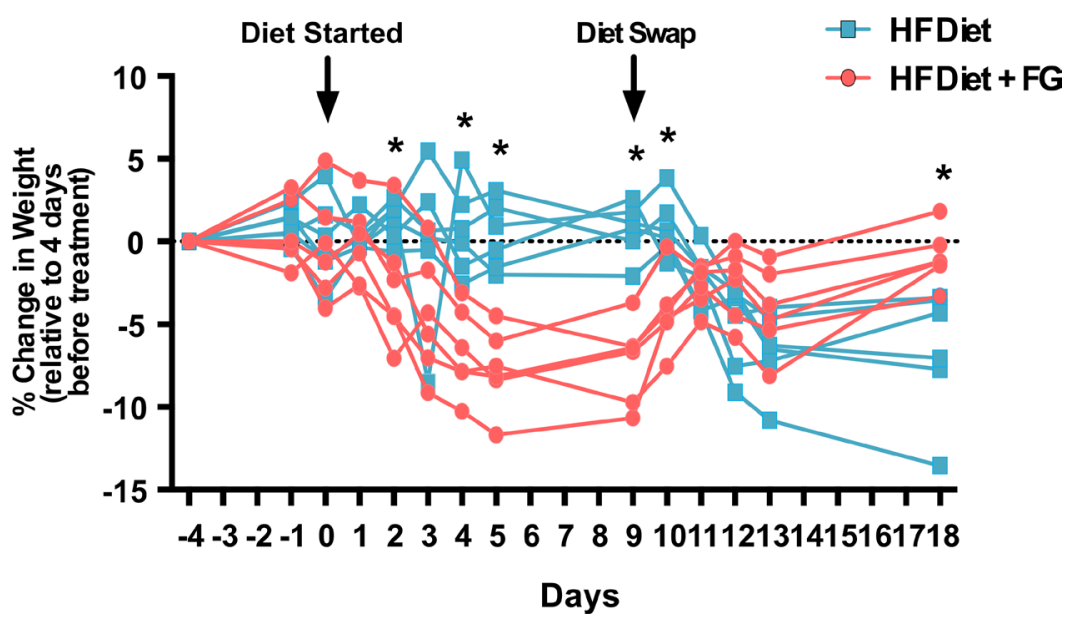

Figure 9. Cumulative change in body weight in germ-free mice after fumagillin administration. Germ-free mice were provided with $\mathrm{HF}$ diet ad libitum from day -4 to -1 . Thereafter, mice received either HF or HF/FG diets ad libitum from day $0-8$. The 2 diets were then switched from day $9-18$. Two-tailed, unpaired $t$ tests were performed. $P<0.05$ was used to define statistical significance among groups. ${ }^{*} P<0.05$ between the 2 groups. $n=6 / g r o u p$.

body weight and aggressive feeding behaviors in patients with Prader-Willi syndrome, a condition characterized by extreme hunger, low metabolic rate, and profound, life-threatening obesity (10).

Metabolic cage studies revealed several physiologic changes in HF/FG compared with HF/PF rats. The aggressive feeding behavior of $\mathrm{HF} / \mathrm{PF}$ rats was reflected in sharp increases in $\mathrm{VO}_{2}$, heat production, physical activity, and RER immediately after provision of food at ZT6:00 (1 pm) in our once-daily feeding regimen. In addition, aggregate measurements over 24 hours revealed clear and sustained increases in $\mathrm{VO}_{2}$, heat production, and physical activity but not RER in HF/PF compared with HF/FG mice. Thus, despite the larger and more sustained weight loss in HF/FG rats, physical activity and energy expenditure were decreased compared with the HF/PF group. In contrast to the effects of caloric restriction in HF/PF rats to trigger increases in food-seeking behavior and physical activity resulting in increased energy expenditure, fumagillin acts in the presence of the same degree of caloric restriction to conserve energy.

We identified multiple metabolic differences between the HF/FG and HF/PF groups, many of which are likely driven by the change in feeding pattern and a more pronounced extent of fasting in HF/PF animals. Differences in liver glycogen and methionine levels were investigated further, given that prior studies had implicated both in regulation of feeding behaviors (19-21). However, neither maintenance of elevated liver glycogen levels by recombinant adenovirus-mediated engineering, dietary methionine restriction, nor both simultaneously, were able to suppress the aggressive feeding behavior of HF/PF rats.

Consistent with its effects on multiple measures of energy expenditure, fumagillin blunts sympathetic tone in the HF/FG compared with the HF/PF group, as evidenced by a clear decrease in urine catecholamines. Also consistent with a profile of energy conservation, body temperatures were decreased at multiple time points in both the dark and light cycles in HF/FG compared with HF/PF rats. Finally, an array of genes associated with torpor-like states, including Cel, Clps, Pnlip, and Pnliprp2, trended to be induced by fumagillin feeding. A torpor-like phenotype has previously been reported in response to FGF21 administration in mice (14); here, we also observed an increase in FGF21 expression in liver of HF/FG rats, consistent with increased plasma levels of FGF21 in obese patients treated with the fumagillin analog beloranib (9).

In light of the surprising finding of a larger and more sustained degree of weight loss in HF/FG compared with HF/PF rats, despite the equally reduced food intake and lower energy expenditure observed in the former group, we investigated potential contributions of altered gastrointestinal function or activities of the gut microbiota in mediating these effects. We found no evidence of differences in gastric emptying or gastrointestinal absorptive function in HF/FG compared with HF/PF rats, but analysis of gross fecal heat capture by bomb calorimetry revealed that HF/FG rats retained significantly more energy in their feces than either the HF or HF/PF groups, suggesting that fumagillin alters gastrointestinal energy extraction/absorption, contributing to negative energy balance. This finding was complemented by the observed increase in levels of urinary amino acids in HF/FG compared with HF/PF groups, suggestive of calorie "spilling." 
We also found that, compared with the HF/PF group, the HF/FG group had significantly higher levels of GLP-1 and lower levels of ghrelin - hormones whose production are affected by SCFA produced by gut microbial fermentation $(17,18)$. The increase in the GLP-1/ghrelin ratio in HF/FG rats is consistent with the observed changes in feeding behavior in these animals. These observations led us to test if fumagillin-induced changes in the functional configuration of the gut microbiota were causally related to feeding behavior and energy balance. Transplantation of cecal microbiota from HF/FG or HF/PF rats to germ-free mice revealed no differences in total food intake or feeding behavior when the mice were fed ad libitum. Furthermore, the 2 groups exhibited the same transition to aggressive feeding behavior when subjected to caloric restriction. Consistent with a lack of effect of the HF/FG versus HF/PF microbiota on feeding behavior, we found that feeding of germ-free mice with HF/FG diet was sufficient to reduce food intake. Also aligning with these findings, liver circadian gene expression was not different between mice colonized with microbiota from $\mathrm{HF} / \mathrm{FG}$ and HF/PF rats, indicating that the ability of fumagillin to block programming of these genes in response to caloric restriction is not mediated by effects on the gut microbiota. In contrast, induction of torpor genes was observed in mice colonized with cecal contents from HF/FG, but not HF/PF, rats. Only modest differences were noted in the structural configuration of the microbiota of recipient mice characterized in the current study. Future studies of the microbiota of larger numbers of rats subjected to the various dietary manipulations described (including comprehensive analyses of the organismal and gene content of their gut communities, microbial gene expression, and expressed microbial metabolic activities) will be needed to better understand the effects of fumagillin. The same multi-omics approach should also be applied to gnotobiotic mouse recipients of microbiota from multiple rats from each of the treatment groups, and the results correlated with metabolic cage studies, although performing this latter type of phenotypic analysis is technically challenging when mice reside in gnotobiotic isolators. Nonetheless, our current results suggest that the effect of the microbiota to regulate torpor genes may contribute to or serve as a marker of energy conservation during fumagillin feeding.

In sum, these studies define a set of physiological and behavioral responses to fumagillin that contribute to a larger and more sustained weight loss response than achieved with caloric restriction alone. Given the very high rates of recidivism typically observed in obese humans that attempt to engage in caloric restriction, an implication of our findings is that more sustained and robust weight loss intervention strategies may emerge from further analysis of the biological effects of fumagillin and related compounds.

\section{Methods}

Supplemental Methods are available online with this article.

Animals and dietary regimens. Male Wistar rats (150-175 g, 6 weeks of age, Charles River Laboratories) were singly housed under a 12-hour/12-hour light/dark cycle (lights on at $7 \mathrm{am}$, also referred to as ZT0:00) with free access to water and fed a HF diet or a plant polysaccharide-based SC diet (Envio, catalog 7001) for 12 weeks prior to further dietary interventions (Supplemental Table 1). Food intake and body weights were monitored weekly during this period. Animals were then subjected to 2, 4, or 8 weeks of the following dietary interventions: (i) HF group; (ii) HF/FG group; (iii) HF/PF group, or (iv) SC group. Food intake and body weights were monitored daily at ZT6:00 (1 pm); food was provided to each group once daily immediately after weighing at ZT6:00 (1 pm).

The dicyclohexylamine salt of fumagillin was purchased from Medivet. This material was dissolved in 1:6 water/ethyl acetate and washed 3 times with $1 \mathrm{~N}$ phosphoric acid (MedChem Partners). The resulting organic solution was treated with activated carbon and sodium sulfite. The solids were then removed by filtration, and the material was condensed on a rotovap (Büchi R-205). The resulting solids were titrated with ethyl acetate to yield fumagillin free acid ( $98.1 \%$ purity by LC/MS). The average weight of rats throughout this study was $529 \mathrm{~g}$, and given a dose of $80 \mathrm{mg}$ fumagillin/ $\mathrm{kg}$ food and an average HF/FG food intake of $13.8 \mathrm{~g} /$ day, the average daily dose of fumagillin was 2.08 grams of fumagillin per rat per day.

Multiple cohorts were subjected to these feeding protocols and used for the following studies. At the end of the intervention periods, one cohort was sacrificed for collection of plasma, liver, gastrocnemius muscle, and adipose tissue after i.p. injection of Nembutal at a dose of $250 \mathrm{mg} / \mathrm{kg}$ body weight. All dissected tissue/organs were rinsed with chilled saline, dried on gauze, and weighed quickly, followed by clamp freezing. All biospecimens were stored at $-80^{\circ} \mathrm{C}$ for hormone, metabolomics, or gut microbiota analyses. A second cohort of rats were analyzed by indirect calorimetry and physical activity measurements in metabolic cages for 40 hours, with continued provision of their respective diets. They were then returned to their 
home cages for 1 week, followed by analysis of insulin sensitivity by hyperinsulinemic-euglycemic clamp (24). The third and fourth cohorts were subjected to 4 weeks of dietary intervention and used for measurement of rectal temperature and additional tissue collection. The fifth cohort of animals only included HF/ FG and HF/PF groups and were used for measurement of gastric emptying. The sixth cohort of animals was fed with either HF or HF/MR diets for a 5-week intervention that included treatment with various recombinant adenoviruses over the last 7 days. Finally, several cohorts were used for feeding behavior studies, with food intake monitored at ZT6:00, ZT10:00, ZT14:00, and ZT2:00 (1 pm, 5 pm, 9 pm, and 9 am) daily over multiple days.

Hyperinsulinemic-euglycemic clamps. Insulin sensitivity was measured by hyperinsulinemic-euglycemic clamps using recently described methods (24). Clamps were performed in conscious unrestrained animals using swivels and tethers (Instech Laboratories) to allow uninterrupted movement of the animals without disruption of infusion lines. Hyperinsulinemia was achieved by a primed i.v. infusion (720 mU/ $\mathrm{kg} / \mathrm{min}$ for 7 minutes) followed by continuous infusion ( $4 \mathrm{mU} / \mathrm{kg} / \mathrm{min}$ ). Blood was sampled from arterial lines at 5-minute intervals, and glucose levels were quantified (BD Logic Glucose Monitor). Euglycemia, defined as blood glucose above $10 \%$ of individual fasting glycemia, was restored and maintained by variable infusion of $30 \%$ dextrose. Steady state was achieved approximately 90 minutes after initiating hyperinsulinemia and maintained for at least 45 minutes. Rats were euthanized immediately following the clamp procedure.

Plasma and tissue metabolomics analyses. Analyses of plasma acylcarnitines, amino acids, conventional metabolites, and hormones, as well as liver and gastrocnemius muscle acylcarnitines, acyl CoAs, ceramides, amino acids, and organic acids, were performed as described previously (24-27).

Indirect calorimetry. Indirect calorimetry was performed using an 8-chamber comprehensive lab animal monitoring system (CLAMS) (Columbus Instruments). Rats fed on the various diets described above were acclimatized to the system overnight prior to measurements collected over a 48-hour period. Food was provided to all experimental groups of rats at ZT6:00 (1 pm) daily, and the light cycle was set from ZT0:00 (7:00 am) to ZT12:00 (7:00 pm).

Quantitative PCR. Stomach, sections of intestine, liver, and adipose tissue were collected, snap-frozen and stored at $-80^{\circ} \mathrm{C}$. RNA was harvested using the RNeasy minikit (Qiagen), and cDNA was synthesized with the iScript reaction (Bio-Rad). Real-time PCR was performed using the ViiA7 sequence detection system and software (TaqMan-based Assay on Demand; Applied Biosystems). Sequences of all primers used for real-time PCR in the study are provided in Supplemental Table 4.

Bomb calorimetry. Fecal samples and pulverized diets were freeze-dried at $-35^{\circ} \mathrm{C}$ for 48 hours using a vacuum freeze-dry chamber (Labconco, catalog 7400040, FreeZone Triad Freeze Dry Systems). Samples were stored under a vacuum at $-80^{\circ} \mathrm{C}$, and energy content was measured using a semi-micron oxygen bomb calorimeter, calorimetric thermometer, and semi-micro oxygen bomb (models 6725, 6772, and 1109A, respectively, from Parr Instrument Co.). The energy equivalent (EE) factor for the calorimeter was calculated using benzoic acid standards.

Measurement of gastric emptying. Acetaminophen absorption and gastric emptying experiments were performed as described previously (16), with some modifications. Groups of HF/FG and HF/PF rats were followed for 4 weeks. We controlled for the fact that HF/PF rats consumed food more rapidly than members of the HF/FG treatment group by providing food for the final 2 days before the study at ZT12:00 $(7 \mathrm{pm})$ rather than ZT6:00 (1 pm) (to shorten the feeding cycle). After the 2-day conditioning period, food was withdrawn overnight from the HF/FG group, followed by ad libitum refeeding with the HF/FG diet from ZT2:00-4:00 (9-11 am) the following morning. The HF/PF group was subjected to the same protocol with 1-day offset to allow food intake to be measured for the 2-hour refeeding period in the HF/FG group. The amount of food consumed by the HF/FG group was measured, and the same amount of HF diet was provided to the $\mathrm{HF} / \mathrm{PF}$ group during its refeeding period. Both groups of rats were sacrificed after their 2-hour refeeding period for measurement of residual food in their stomachs. After stomach content was retrieved and dried at $50^{\circ} \mathrm{C}$ for 72 hours, the percentage of food content remaining in the stomach was determined from the dry weight of stomach content divided by the total amount of food consumed per animal, multiplied by 100 (16).

Measurement of tissue glycogen and GSK3 $\beta$. Tissue glycogen content was measured as described previously (25). Levels of phosphorylated GSK3 $\beta$ were determined using a commercially available kit (Meso Scale Discovery; catalog K150CQD-1 for phosphorylated GSK3 $\beta$ and catalog K150CRD-1 for total GSK3 $\beta$ ). 
Infusion of recombinant adenoviruses and immunoblotting. Recombinant adenoviruses $\left(2 \times 10^{9} \mathrm{particle} / \mathrm{g}\right.$ body weight) were infused via tail vein in isoflurane-anesthetized animals. Cyclosporine A (Bedford Laboratories) was given i.p. at a dose of $15 \mathrm{mg} / \mathrm{kg}$ on the day before and the day of adenovirus infusions (25). Construction and use of recombinant adenoviruses containing a modified version of the muscle isoform of AdCMV-GM $\triangle \mathrm{C}$ and AdCMV- $\beta$ Gal have been described previously (21).

Immunoblot analyses were performed using Bio-Rad Criterion TGX gels (catalog 567-1083), according to the protein blotting guide provided by Bio-Rad. Anti-Flag M2-HRP (catalog A8592) was purchased from MilliporeSigma.

Bacterial $16 S$ rRNA analysis. DNA was isolated from fecal pellets and cecal contents that had been collected from rats or mice by bead-beating in $2 \times$ buffer $\mathrm{A}(200 \mathrm{mM} \mathrm{NaCl}, 200 \mathrm{mM}$ Tris, $20 \mathrm{mM}$ EDTA) and phenol/chloroform/isoamyl alcohol, followed by further purification (QiaQuick 96 purification kit; Qiagen). PCR amplification of the V4 region of bacterial 16S rRNA genes was performed as described (28). Amplicons with sample-specific bar codes were pooled for multiplex sequencing with an Illumina MiSeq instrument. Reads were demultiplexed and rarefied to 5,000 reads/sample. Reads sharing $\geq 97 \%$ nucleotide sequence identity (97\%ID) with a reference sequence in the GreenGenes 16S rRNA database (http://qiime.org/home_static/dataFiles.html) (29) were assigned to that OTU. Unassigned sequences were grouped into de novo 97\%ID OTUs. A phylogenetic tree based on the V4-16S rRNA sequences was constructed. Indicator species analysis was performed in the statistical software package R v.3.2.4 (30) using "indicspecies" (22) to identify 97\%ID OTUs that differed in their representation in the microbiota of gnotobiotic mice colonized with cecal microbial communities from HF/FG versus $\mathrm{HF} / \mathrm{PF}$ rats. Significance was determined by 10,000 permutations, and $P$ values were corrected using the Benjamini-Hochberg method. A second indicator species analysis was performed using the subset of OTUs identified in the mice to determine whether any of them was significantly associated with the fumagillin-treated or pair-fed rats.

Statistics. For plasma and tissue metabolomics analyses, pairwise comparisons were made with a Bonferroni correction, focusing on the differences in mean values of each metabolite between intervention groups. For other physiological analyses in both rat and mouse experiments, unless mentioned otherwise, 2-tailed, unpaired $t$ tests were performed. $P<0.05$ was used to define statistical significance between 2 groups, while $P<0.05$ with a Bonferroni correction was used to define statistical significance in group comparisons involving more than 2 groups.

Study approval. All procedures involving rats were approved by the Duke University IACUC (Duke University School of Medicine). All experiments involving mice were performed using protocols approved by the Washington University Animal Studies Committee (Washington University School of Medicine). Details of protocols used for gnotobiotic mouse experiments are provided in Supplemental Methods.

\section{Author contributions}

CBN, JIG, TEH, JEV, DM, and SAS conceived the study. CBN, JIG, TEH, JA, and MLP designed the study and wrote the manuscript. JA, LW, JMH, and ELG performed rat experiments. JA, LW, and MLP performed mouse experiments. RDS, OI, JRB, and MJM performed metabolomics analyses. ST, JA, VKR, and MLP performed statistical analyses.

\section{Acknowledgments}

Our studies were supported by NIH grants P01DK78669 (to JIG and CBN) and P01DK58398 (to CBN) and a pilot grant from the Duke-NUS Singapore Medical School (to SS, DM, and CBN). We thank David O'Donnell and Maria Karlsson for their help with gnotobiotic animal husbandry; Helena Winfield, Huaxia Cui, Rama Arumugam, and April Wittmann for excellent technical support; and Nicholas Griffin for his input regarding indicator species analysis.

Address correspondence to: Christopher B. Newgard, Duke Molecular Physiology Institute, 300 North Duke Street, Durham, North Carolina 27701, USA. Phone: 919.668.6059; Email: Chris.newgard@duke.edu.

VKR's present address is: Verily Life Sciences, San Francisco, California, USA. 
1. Sumithran P, et al. Long-term persistence of hormonal adaptations to weight loss. N Engl J Med. 2011;365(17):1597-1604.

2. Yanovski SZ, Yanovski JA. Long-term drug treatment for obesity: a systematic and clinical review. JAMA. 2014;311(1):74-86.

3. Abdeen G, le Roux CW. Mechanism Underlying the Weight Loss and Complications of Roux-en-Y Gastric Bypass. Review. Obes Surg. 2016;26(2):410-421.

4. Molina JM, et al. Fumagillin treatment of intestinal microsporidiosis. N Engl J Med. 2002;346(25):1963-1969.

5. Ingber D, et al. Synthetic analogues of fumagillin that inhibit angiogenesis and suppress tumour growth. Nature. 1990;348(6301):555-557.

6. Sin N, Meng L, Wang MOW, Wen JJ, Bornmann WG, Crews GM. The anti-angiogenic agent fumagillin covalently binds and inhibits the methionine aminopeptidase, MetAP-2. Proc Natl Acad Sci U S A. 1997;94(12):6099-6103.

7. Lijnen HR, Frederix L, Van Hoef B. Fumagillin reduces adipose tissue formation in murine models of nutritionally induced obesity. Obesity (Silver Spring). 2010;18(12):2241-2246.

8. Datta B, Majumdar A, Datta R, Balusu R. Treatment of cells with the angiogenic inhibitor fumagillin results in increased stability of eukaryotic initiation factor 2-associated glycoprotein, p67, and reduced phosphorylation of extracellular signal-regulated kinases. Biochemistry. 2004;43(46):14821-14831.

9. Hughes TE, Kim DD, Marjason J, Proietto J, Whitehead JP, Vath JE. Ascending dose-controlled trial of beloranib, a novel obesity treatment for safety, tolerability, and weight loss in obese women. Obesity (Silver Spring). 2013;21(9):1782-1788.

10. McCandless SE, et al. Effects of MetAP2 inhibition on hyperphagia and body weight in Prader-Willi syndrome: A randomized, double-blind, placebo-controlled trial. Diabetes Obes Metab. 2017;19(12):1751-1761.

11. Stephan FK. The "other" circadian system: food as a Zeitgeber. J Biol Rhythms. 2002;17(4):284-292.

12. Hoogerwerf WA, et al. Clock gene expression in the murine gastrointestinal tract: endogenous rhythmicity and effects of a feeding regimen. Gastroenterology. 2007;133(4):1250-1260.

13. Zvonic S, et al. Characterization of peripheral circadian clocks in adipose tissues. Diabetes. 2006;55(4):962-970.

14. Inagaki T, et al. Endocrine regulation of the fasting response by PPARalpha-mediated induction of fibroblast growth factor 21. Cell Metab. 2007;5(6):415-425.

15. Badman MK, Pissios P, Kennedy AR, Koukos G, Flier JS, Maratos-Flier E. Hepatic fibroblast growth factor 21 is regulated by PPARalpha and is a key mediator of hepatic lipid metabolism in ketotic states. Cell Metab. 2007;5(6):426-437.

16. Stephens SB, et al. A VGF-derived peptide attenuates development of type 2 diabetes via enhancement of islet $\beta$-cell survival and function. Cell Metab. 2012;16(1):33-43.

17. Greiner TU, Bäckhed F. Microbial regulation of GLP-1 and L-cell biology. Mol Metab. 2016;5(9):753-758

18. den Besten G, van Eunen K, Groen AK, Venema K, Reijngoud DJ, Bakker BM. The role of short-chain fatty acids in the interplay between diet, gut microbiota, and host energy metabolism. J Lipid Res. 2013;54(9):2325-2340.

19. Hopkins M, Jeukendrup A, King NA, Blundell JE. The relationship between substrate metabolism, exercise and appetite control: does glycogen availability influence the motivation to eat, energy intake or food choice? Sports Med. 2011;41(6):507-521.

20. Harper AE, Benevenga NJ, Wohlhueter RM. Effects of ingestion of disproportionate amounts of amino acids. Physiol Rev. 1970;50(3):428-558

21. Yang R, Cao L, Gasa R, Brady MJ, Sherry AD, Newgard CB. Glycogen-targeting subunits and glucokinase differentially affect pathways of glycogen metabolism and their regulation in hepatocytes. J Biol Chem. 2002;277(2):1514-1523.

22. De Cáceres M, Legendre P. Associations between species and groups of sites: indices and statistical inference. Ecology. 2009;90(12):3566-3574.

23. Dufrêne M, Legendre P. Species assemblages and indicator species: the need for a flexible asymmetrical approach. Ecol Monogr. 1997;67(3):345-366

24. White PJ, et al. Branched-chain amino acid restriction in Zucker-fatty rats improves muscle insulin sensitivity by enhancing efficiency of fatty acid oxidation and acyl-glycine export. Mol Metab. 2016;5(7):538-551

25. An J, et al. Hepatic expression of malonyl-CoA decarboxylase reverses muscle, liver and whole-animal insulin resistance. Nat Med. 2004;10(3):268-274.

26. Newgard CB, et al. A branched-chain amino acid-related metabolic signature that differentiates obese and lean humans and contributes to insulin resistance. Cell Metab. 2009;9(4):311-326.

27. Siddique MM, et al. Ablation of dihydroceramide desaturase 1, a therapeutic target for the treatment of metabolic diseases, simultaneously stimulates anabolic and catabolic signaling. Mol Cell Biol. 2013;33(11):2353-2369.

28. Bokulich NA, et al. Quality-filtering vastly improves diversity estimates from Illumina amplicon sequencing. Nat Methods. 2013;10(1):57-59.

29. DeSantis TZ, et al. Greengenes, a chimera-checked 16S rRNA gene database and workbench compatible with ARB. Appl Environ Microbiol. 2006;72(7):5069-5072.

30. R Core Team. R: A language and environment for statistical computing. R Foundation for Statistical Computing, Vienna, Austria. http://www.R-project.org/. Accessed February 20, 2018 